\title{
COVID-19 and Antiphospholipid Antibodies: Time for a Reality Check?
}

\author{
Emmanuel J. Favaloro, PhD, FFSc, (RCPA) ${ }^{1,2(0)}$ Brandon Michael Henry, MD ${ }^{3}$ Giuseppe Lippi, MD $4(0)$
}

1 Department of Haematology, Sydney Centres for Thrombosis and Haemostasis, Institute of Clinical Pathology and Medical Research (ICPMR), NSW Health Pathology, Westmead Hospital, Westmead, NSW, Australia

${ }^{2}$ School of Biomedical Sciences, Charles Sturt University, Wagga Wagga, NSW, Australia

${ }^{3}$ Cardiac Intensive Care Unit, The Heart Institute, Cincinnati

Children's Hospital Medical Center, Cincinnati, Ohio

${ }^{4}$ Section of Clinical Biochemistry, University of Verona, Verona, Italy

Semin Thromb Hemost 2022;48:72-92.

\begin{abstract}
Address for correspondence Emmanuel J. Favaloro, PhD, FFSc (RCPA) Department of Haematology, Sydney Centres for Thrombosis and Haemostasis, Institute of Clinical Pathology and Medical Research (ICPMR), NSW Health Pathology, Westmead Hospital, Westmead, NSW, 2145 Australia

(e-mail: emmanuel.favaloro@health.nsw.gov.au).
\end{abstract}

\author{
Abstract \\ Keywords \\ - antiphospholipid \\ antibodies \\ - anticardiolipin \\ antibodies \\ - anti-ß2-glycoprotein I \\ antibodies \\ - lupus anticoagulant \\ - COVID-19 \\ - microthrombosis \\ - thrombosis
}

Antiphospholipid antibodies ( $\mathrm{aPL}$ ) comprise a panel of autoantibodies that reflect a potential prothrombotic risk in several autoimmune conditions, most notably antiphospholipid (antibody) syndrome (APS). aPL can be divided into those that form part of the laboratory criteria for APS, namely, lupus anticoagulant (LA), as well as anticardiolipin antibodies (aCL) and anti- 32 -glycoprotein I antibodies (aß2GPI) of the immunoglobulin $\mathrm{G}$ and $\mathrm{M}$ classes, and those that form a group considered as "noncriteria antibodies." The noncriteria antibodies include, for example, antiphosphatidylserine antibodies (aPS), antiprothrombin antibodies (aPT), and antiphosphatidylserine/prothrombin complex antibodies (aPS/PT). COVID-19 (coronavirus disease 2019) represents a prothrombotic disorder, and there have been several reports of various aPL being present in COVID-19 patients. There have also been similarities drawn between some of the pathophysiological features of COVID-19 and APS, in particular, the most severe form, catastrophic APS (CAPS). In this review, we critically appraise the literature on aPL and COVID-19. This is a companion piece to a separate review focused on LA. In the current review, we primarily concentrate on the so-called solid phase identifiable aPL, such as $\mathrm{aCL}$ and aß2GPI, but also reflect on noncriteria aPL. We conclude that aPL positivity may be a feature of COVID-19, at least in some patients, but in general, identified "solid-phase" aPL are of low titer and not able to be well-linked to the thrombotic aspects of COVID-19. Also, most publications did not assess for aPL persistence, and where persistence was checked, the findings appeared to represent transient aPL. Importantly, high-titer aPL or multiple aPL positivity (including double, triple) were in the minority of COVID-19 presentations, and thus discount any widespread presence of APS, including the most severe form CAPS, in COVID-19 patients. published online June 15, 2021
Issue Theme Maintaining Hemostasis and Preventing Thrombosis in COVID-19-Part III; Guest Editors: Emmanuel J. Favaloro, PhD, FFSc (RCPA) and Giuseppi Lippi, MD (c) 2021. Thieme. All rights reserved. Thieme Medical Publishers, Inc., 333 Seventh Avenue, 18th Floor, New York, NY 10001, USA
DOI https://doi.org/ 10.1055/s-0041-1728832. ISSN 0094-6176. 
Antiphospholipid antibodies (aPL) comprise a broad panel of autoantibodies that reflect a potential prothrombotic risk in several autoimmune conditions, most notably antiphospholipid (antibody) syndrome (APS). ${ }^{1,2}$ aPL can be divided into those that form part of the laboratory criteria for APS and those that do not, being the so-called noncriteria antibodies. ${ }^{1,2}$ The aPL forming the laboratory criteria for APS comprise lupus anticoagulant (LA), as well as anticardiolipin antibodies ( $\mathrm{aCL}$ ) and anti- $\beta 2$-glycoprotein I antibodies (aß2GPI) of the immunoglobulin (Ig) G and $\mathrm{M}$ classes. ${ }^{1,2}$ The remaining aPL can thus be considered to form another group of aPL and alternatively defined as "noncriteria" antibodies. This latter class of antibodies comprises higher numbers of aPL types, and include, for example, any Ig class of antiphosphatidylserine antibodies (aPS), antiprothrombin antibodies (aPT), antiphosphatidylserine/prothrombin complex antibodies (aPS/PT), antiphosphatidylinositol antibodies, antiannexin $\mathrm{V}$ antibodies, and anti- $\beta 2 \mathrm{GPI}$-domain 1 antibodies, and also aCL and aß2GPI of IgA class.

To be identified as having APS, there is a requirement to show evidence of at least one of the laboratory criteria (LA, IgG or IgM aCL, or a $32 \mathrm{GPI}$ ), in medium or high titer, as well as their persistence by retesting on a second occasion some 12 weeks later, plus at least one of the clinical criteriathrombosis or pregnancy morbidity. ${ }^{1,2}$

While several authors propose added value of noncriteria $\mathrm{aPL}^{3-6}$ current guidelines suggest insufficient evidence for their current inclusion as "criteria" aPL for APS. ${ }^{2}$ On the other hand, there is also some debate about the value of some of the established criteria aPL, such as aCL IgM and aß2GPI IgM, for identification of APS. ${ }^{7-9}$ In any case, LA appears to represent the entity with greatest relevance to thrombosis risk in APS among all the aPL, ${ }^{10}$ perhaps followed by aß2GPI of IgG class.

COVID-19 (coronavirus disease 2019) is a prothrombotic disorder and there have been several reports of various aPL being present in COVID-19 patients. There have also been similarities drawn between some of the pathophysiological features of COVID-19 and APS, in particular, the most severe form, catastrophic APS (CAPS). In this review, we critically appraise the literature on aPL and COVID-19. This is a companion piece to a separate review focused on LA. ${ }^{11}$ In the current review, we primarily focus on the so-called solid phase identified aPL, such as aCL and aß2GPI, but also reflect on the noncriteria aPL.

\section{COVID-19}

COVID-19 has been declared a pandemic by the World Health Organization (WHO), and is caused by infection with SARSCoV-2 (severe acute respiratory syndrome coronavirus 2). COVID-19 is thought to have originated in Wuhan, China, in late 2019, and at the time of writing has infected over 120 million people and caused nearly 2.7 million deaths. ${ }^{12}$ Severe COVID-19 is first and foremost a prothrombotic disorder, ${ }^{13}$ with thrombosis appearing in various forms. For example, a recent meta-analysis has indicated a venous thrombosis rate, including deep vein thrombosis and pulmonary thrombosis, of close to $30 \%$ in severe COVID-19. ${ }^{14}$ Acute myocardial ischemia (infarction) and cerebrovascular accidents may also develop in as many as 8 and 3\% of COVID-19 patients needing intensive care, ${ }^{15}$ while systemic coagulopathy and disseminated intravascular coagulation may occur in as many as $7 \%$ of such patients. ${ }^{16}$ Evidence of microthrombosis in multiple organs including lungs, kidneys, and liver also occurs, although it is only identifiable on autopsy in patients who have died due to COVID-19. ${ }^{17-20}$

As part of a search to investigate the mechanisms that promote thrombosis in COVID-19, many tests of hemostasis have been investigated in patients suffering from this disease. Indeed, many hemostasis tests are abnormal in patients with COVID-19. ${ }^{21,22}$ Moreover, COVID-19 appears to affect all aspects of hemostasis, including primary hemostasis (endothelium, platelets, von Willebrand factor), secondary hemostasis/coagulation, and fibrinolysis. ${ }^{23-28}$

\section{COVID-19 and APS?}

Relevant to this review is that there have been several reports of similarities between some of the pathophysiological features of COVID-19 and APS, in particular, the most severe form, CAPS. ${ }^{29-31}$ For example, patients with COVID-19 appear to fulfil the main clinical diagnostic criteria for CAPS, with the criteria being evidence of involvement in three or more organs, development of manifestations simultaneously or in less than a week, and confirmation by histopathology of small vessel occlusion in at least one organ. ${ }^{29}$ There have also now been many reports identifying various aPL in COVID-19 patients. The search for aPL in COVID-19 may have been sparked by an early publication by Zhang et $\mathrm{al}^{32}$ in the New England Journal of Medicine.

Given that (1) aPL are associated with thrombosis, (2) patients with COVID-19 suffer thrombosis, (3) some aspects of COVID-19 pathology strongly resemble (catastrophic) APS (CAPS), and (4) aPL have been identified in COVID-19 in several studies, several questions arise, including that of the significance of the aPL in COVID-19, as well as any potential involvement in COVID-19 pathology. Is APS, or indeed CAPS, really a feature of COVID-19? We critically appraise the literature to help address these questions. We, however, note that the current review is a companion piece to a previous related review on LA. ${ }^{11}$ Here, we focus on the socalled solid phase detected aPL.

\section{Thrombosis-Associated aPL versus Laboratory- Detected aPL}

Similar to the previous review on LA, ${ }^{11}$ despite an association of aPL with thrombosis risk in APS and in other potential autoimmune diseases, the presence of a laboratory-detected aPL per se does not, in itself, reflect prothrombotic risk factors, even if persistent, and does not warrant pharmacological intervention in asymptomatic patients, ${ }^{33,34}$ except perhaps for those with high-titer aPL and multiple positivity. ${ }^{35,36}$ Indeed, laboratory-detected aPL may be found in asymptomatic patients, and otherwise reflect chance 
findings. Many of the patients in whom aPL are detected will not develop thrombosis.

\section{aPL Testing Guidelines and Assay Cut-offs}

There are several groups who have provided guidelines on aPL testing. ${ }^{1,2,7-9,37-39}$ These identify not only the various types of aPL tests, but also, in some cases, how they should be performed. For example, the LA guidelines provide advice on performance of clot-based ("liquid phase") tests, and include which tests to perform and also procedural processes on how they should be performed and interpreted. ${ }^{37-39}$ Likewise, guidance for the "solid phase" aPL is also available, including which tests to perform and also procedural processes on how they should be performed and interpreted. ${ }^{1,2,7-9}$ It needs also to be recognized here, however, that manufacturers of aPL assays cannot be mandated to produce assay kits according to these guidelines and that, in reality, a wide range of methodologies and assays may be employed to identify aPL. Thus, although different workers may report on the same apparent aPL (e.g., aCL of IgG class), differences in methods of detection (e.g., enzyme-linked immunosorbent assay [ELISA] vs. chemiluminescence immunoassay [CLIA]) mean that different findings may be reported using different methods for that same aPL. Therefore, variation in literature reporting for any given aPL will reflect a variety of factors, including both a difference in the COVID-19 cohort evaluated and the method employed to detect a particular aPL.

Furthermore, there are differences in how laboratories and manufacturers may assign a cut-off value for defining a positive aPL result. In general, the recommendations indicate the 99th percentile of at least 120 normal individuals, or $>40$ GPL or MPL units. However, some methods and laboratories will assign positivity with lower cut-off values, and thus potentially identify a greater proportion of aPL-positive cases. According to current guidelines, APS is not assigned unless medium to high titers are identified (generally meaning $>40$ GPL or MPL units). ${ }^{1,2}$ Furthermore, many automated methods for solid-phase aPL are now available, including chemiluminescence-based immunoassays (e.g., CLIA on AcuStar/BioFlash). These methods may use alternate units such as arbitrary chemiluminescence units (CU). Interestingly, the manufacturers may have tried to at least partially harmonize cut-off values, which in most cases are around 20 GPL, MPL, or CU. However, other cut-off values may be used, depending on the assay and methodology, such as 8,15 , or 40 GPL, MPL, or CU.

\section{Literature Search}

To give some additional background to this narrative review, we searched the PubMed database (https://pubmed. ncbi.nlm.nih.gov) using various iterations of COVID-19 together with various iterations of LA and (anti)phospholipid antibodies. An initial search performed on February, 22, 2021, was later updated to be current as of March 6, 2021. Of over 200 separate articles identified by this search, we then excluded general reviews, commentaries, and papers otherwise found to be irrelevant to the topic. We also excluded single-case reports, but small case series were included.

\section{Results of the Literature Review: Is aPL Present in COVID-19?}

We have already described the literature on LA in COVID$19 .{ }^{11}$ A summary of the literature arising from our search and related to other (i.e., solid phase identified) aPL is given in -Table 1. Note, however, that some studies reported on both solid-phase aPL and LA, and in some cases did not separately identify findings. Irrespective, as for the case with LA, ${ }^{11}$ there was also a large body of publications related to solid-phase aPL. ${ }^{31,32,40-76}$ Although additional relevant papers are likely available in the literature, the captured articles are sufficient for us to critically review the main literature to date. As for $\mathrm{LA},{ }^{11}$ there was a wide variety of methods employed to identify solid-phase aPL ( - Table 1), but sometimes the methodology was not reported. There was a wide variety also in COVID-19 case numbers and type, including in some reports "severe" COVID-19, using a variety of definitions (i.e., needing mechanical ventilation or intensive care; mortality).

Of interest, solid-phase aPL was not always detected in patients with COVID-19, as some studies clearly reported "no aPL" or very few cases of aPL in their patient cohort (-Table 1). However, most publications instead reported a small or notable proportion of their COVID-19 cohorts as expressing solid-phase aPL, although the incidence rarely approached that identified for LA, in which $>80 \%$ of COVID19 cases were sometimes identified to have LA. ${ }^{11}$ Nevertheless, like the case for LA, there does also seem to be a dichotomy of opinions around the presence or not of solidphase aPL in COVID-19. To put a graphical perspective to the data, - Fig. 1 plots the findings from the literature identified in - Table 1 according to percentage positive for aPL versus number of investigated cases. A statistically relevant pattern cannot be seen. Note, however, that in some publications aPL were described as a composite and thus would also have included LA.

One of the earliest reports on the presence of aPL in COVID-19 was by Zhang et al. ${ }^{32}$ This was a case series report of three patients with COVID-19 in intensive care unit who suffered serious sequalae including multiple infarcts in which aPL were detected. This study no doubt prompted a wider search for aPL in subsequent COVID-19 cohorts, but can be criticized in many ways. First, the methodology used for aPL detection was not identified, nor were the titers of identified aPL (high or low?). Persistence of aPL was also not evaluated. As the study focused on a particular small group of COVID-19 patients, there was also clear patient selection bias. In other words, the study focused on three patients with serious clinical sequalae who also happened to have aPL. There was no evidence of cause or effect. To take a dichotomous perspective, the first paper we identified to report on COVID-19 in this arena was from Yasri and Wiwanitkit, in $2020{ }^{40}$ These investigators used data collected "according to public official report of CDC of Thailand, the second country 
Antiphospholipid Antibodies and COVID-19 Favaloro et al. 75

\begin{tabular}{|c|c|c|c|c|c|c|}
\hline 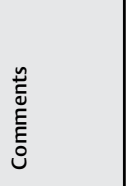 & & & 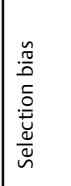 & & 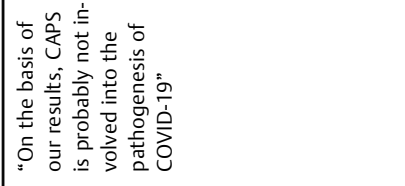 & 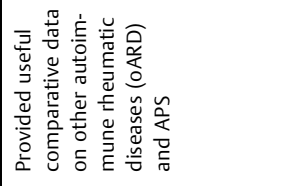 \\
\hline & $\stackrel{\circ}{z}$ & $\stackrel{2}{2}$ & 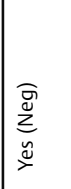 & 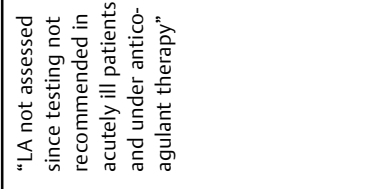 & 2 &  \\
\hline  & $\frac{\alpha}{z}$ & $\frac{\mathscr{a}}{z}$ & 兰 & $\frac{o}{z}$ & $\frac{x}{z}$ & $\frac{o}{z}$ \\
\hline & $\frac{p}{2}$ & 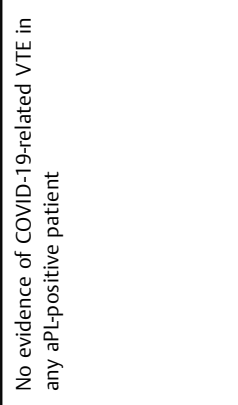 & $\frac{\tilde{z}}{z}$ & 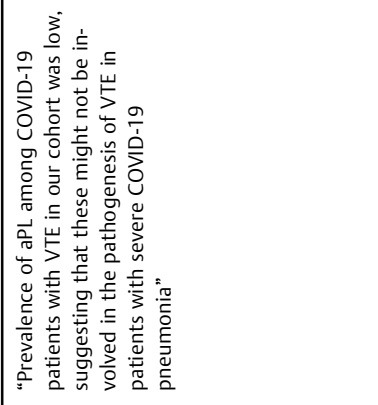 & 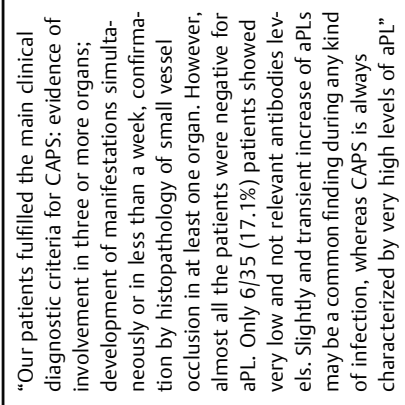 &  \\
\hline 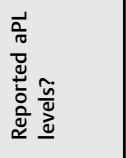 & $\frac{o}{z}$ & $\stackrel{\circ}{z}$ & 妾 & $\stackrel{\check{u}}{\rightleftharpoons}$ & $\stackrel{\tilde{y}}{\rightleftharpoons}$ & $\stackrel{\check{\nu}}{\rightleftharpoons}$ \\
\hline  & 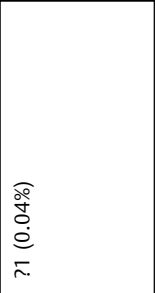 &  & 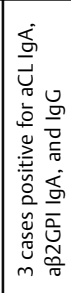 &  & 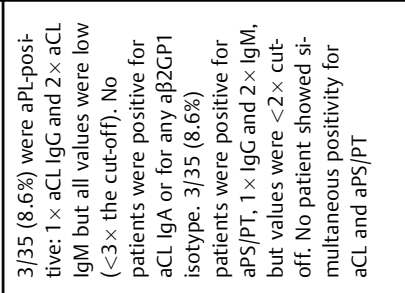 & 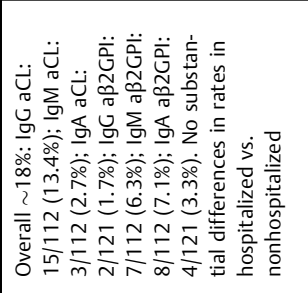 \\
\hline 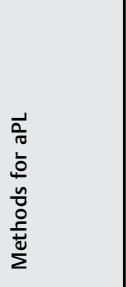 & $\frac{\circ}{z}$ & 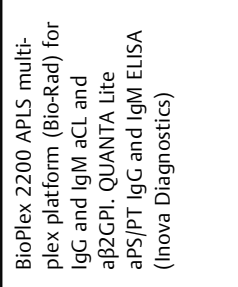 & $\frac{\alpha}{z}$ & 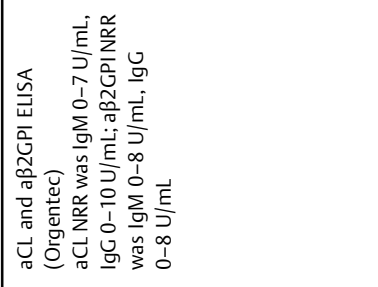 & 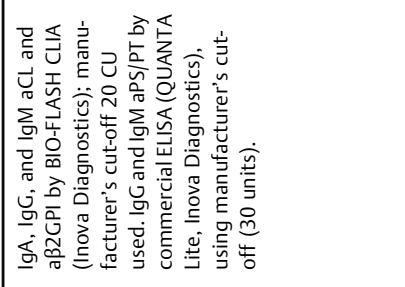 &  \\
\hline 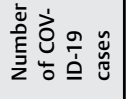 &  & t & $m$ & I & $\stackrel{n}{m}$ & $\tilde{\simeq}$ \\
\hline 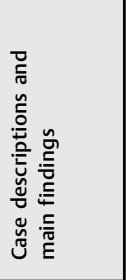 & 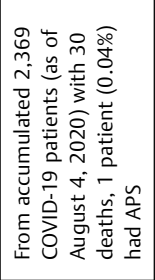 & $\mid \begin{array}{l}\frac{9}{1} \\
\dot{\rho} \\
0 \\
0 \\
0 \\
0\end{array}$ & 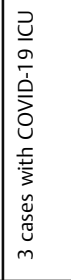 & 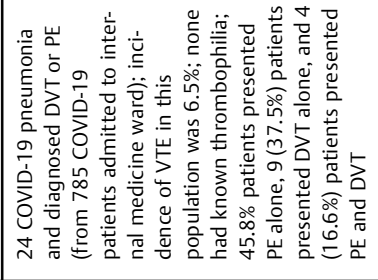 & 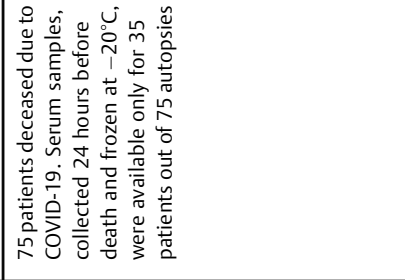 & 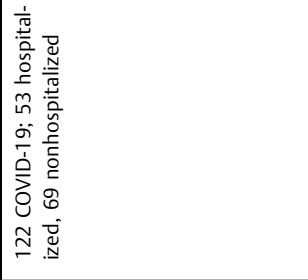 \\
\hline 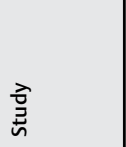 & 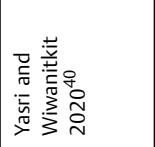 & 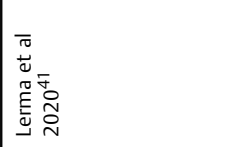 & 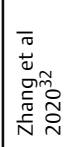 & 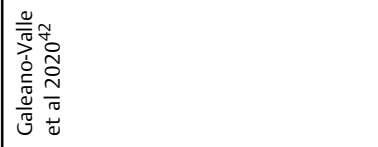 & 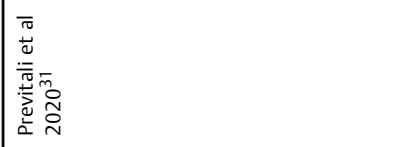 &  \\
\hline
\end{tabular}




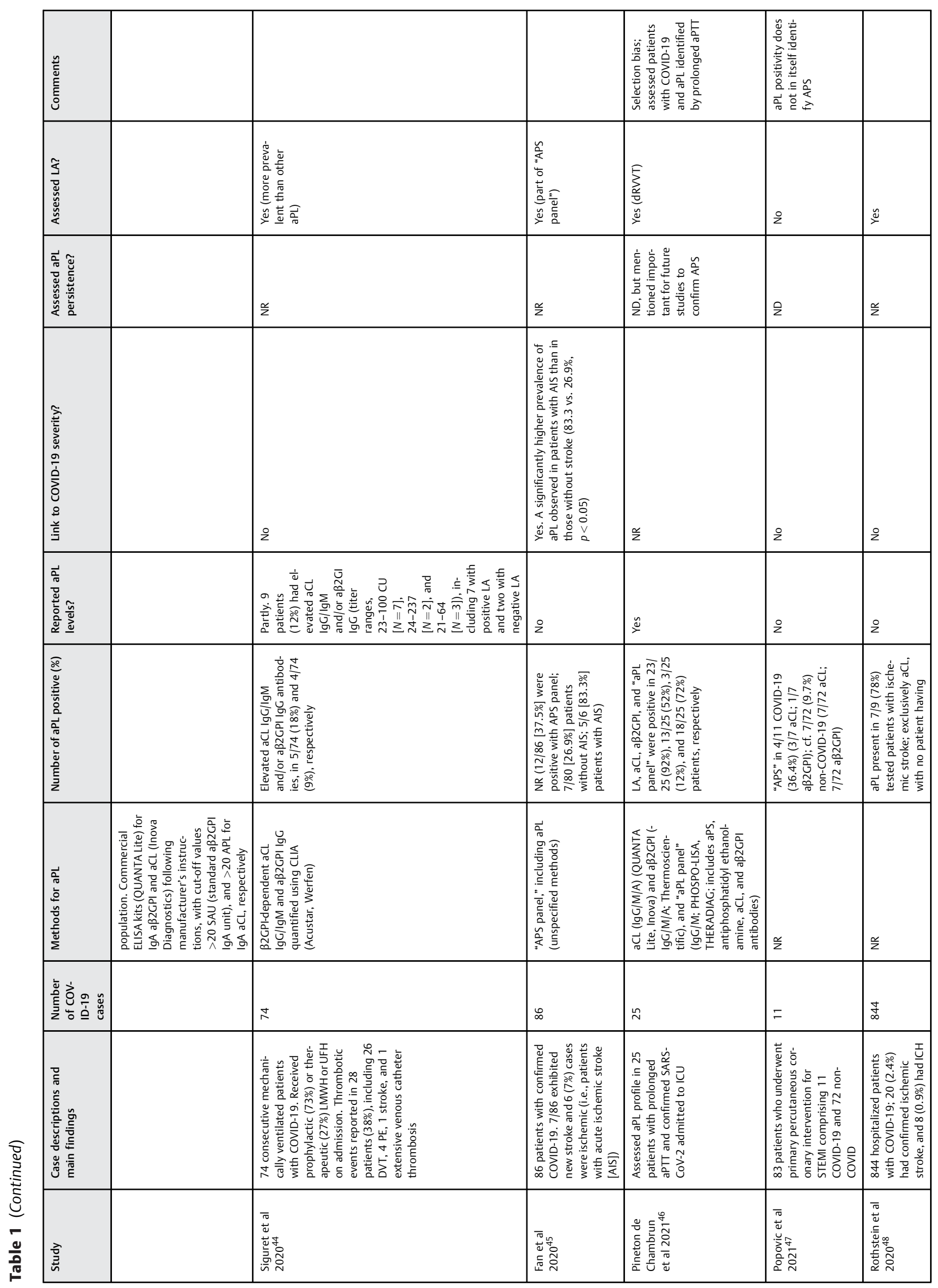




\begin{tabular}{|c|c|c|c|c|}
\hline & & 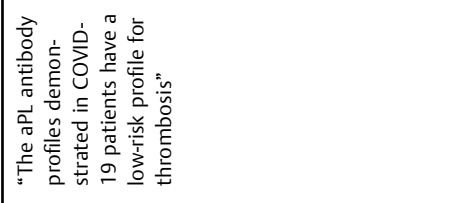 & 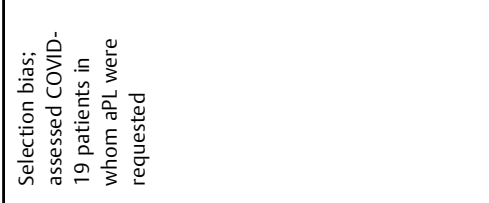 & 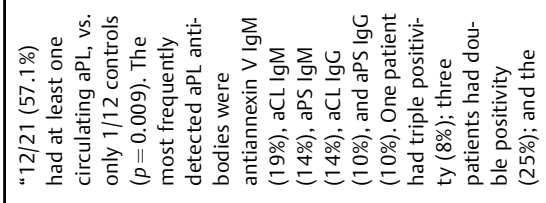 \\
\hline & & 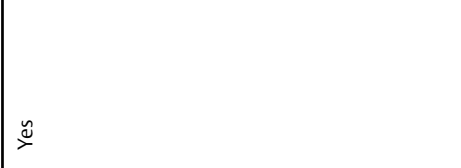 & $\check{\check{\nu}}$ & z \\
\hline 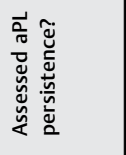 & & 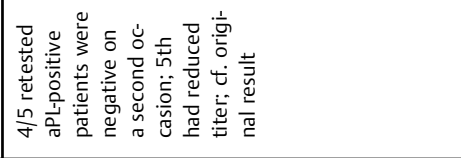 & 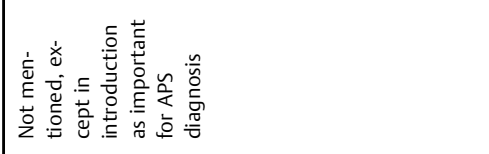 & $\frac{\mathscr{L}}{\Sigma}$ \\
\hline & & 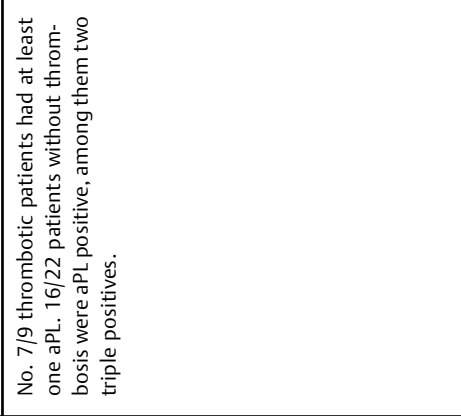 & 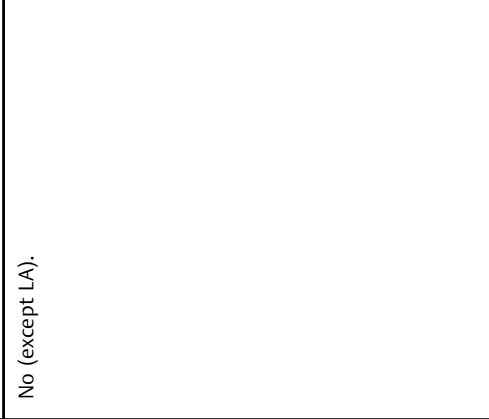 & 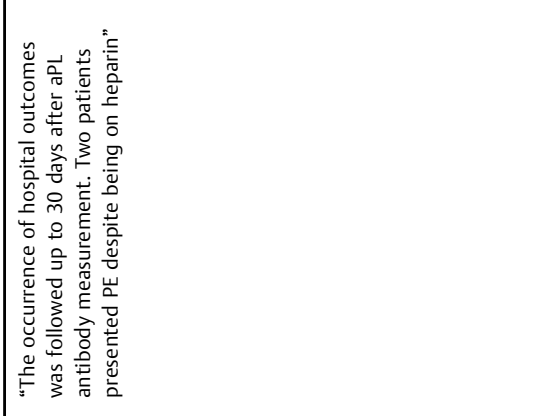 \\
\hline 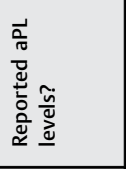 & &  & $\stackrel{\circ}{2}$ & ஜ \\
\hline 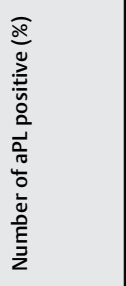 & 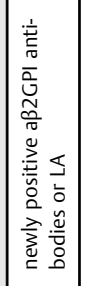 & 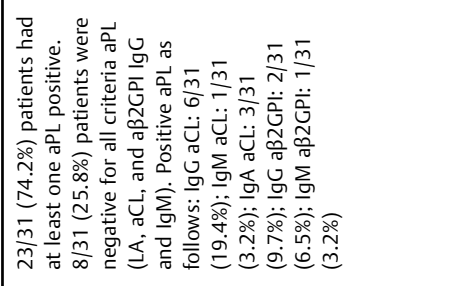 & 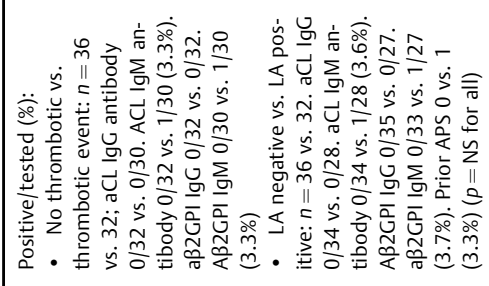 &  \\
\hline 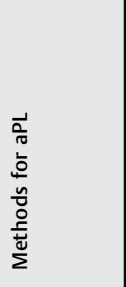 & & 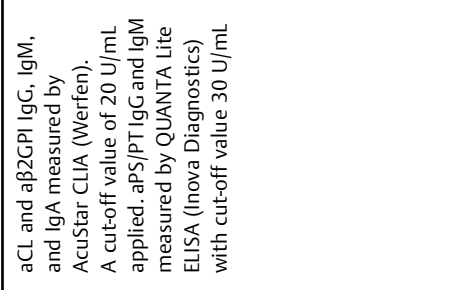 & 兰 & 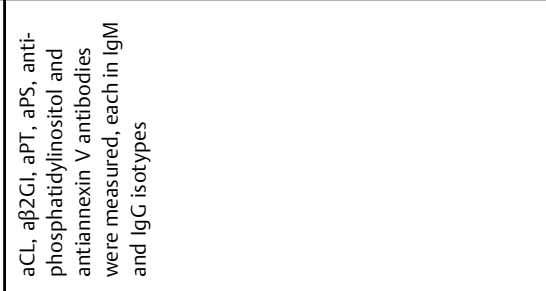 \\
\hline 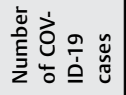 & & $\bar{m}$ & $\infty$ & $\bar{\sim}$ \\
\hline 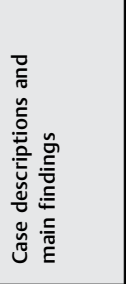 & & 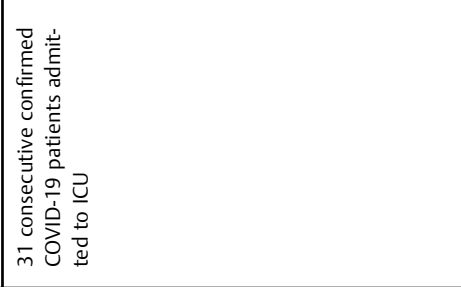 & 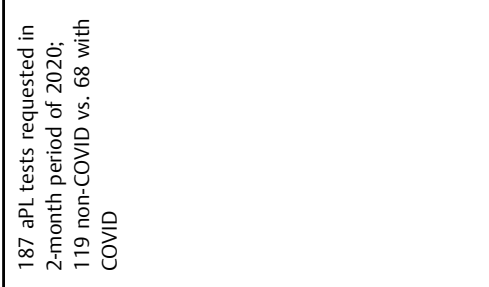 & 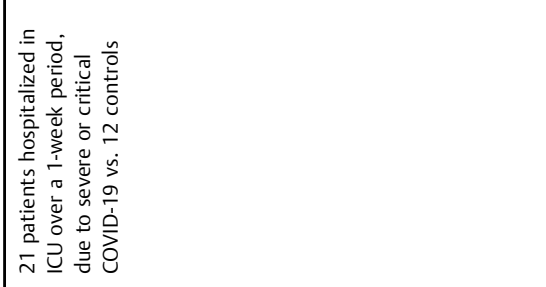 \\
\hline 离 & & 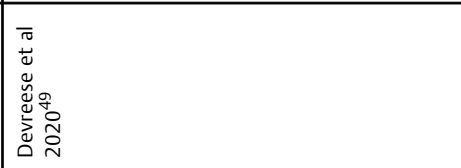 & 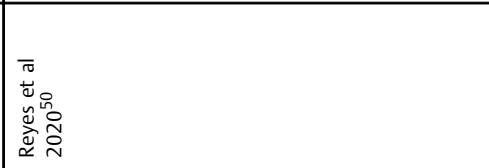 &  \\
\hline
\end{tabular}




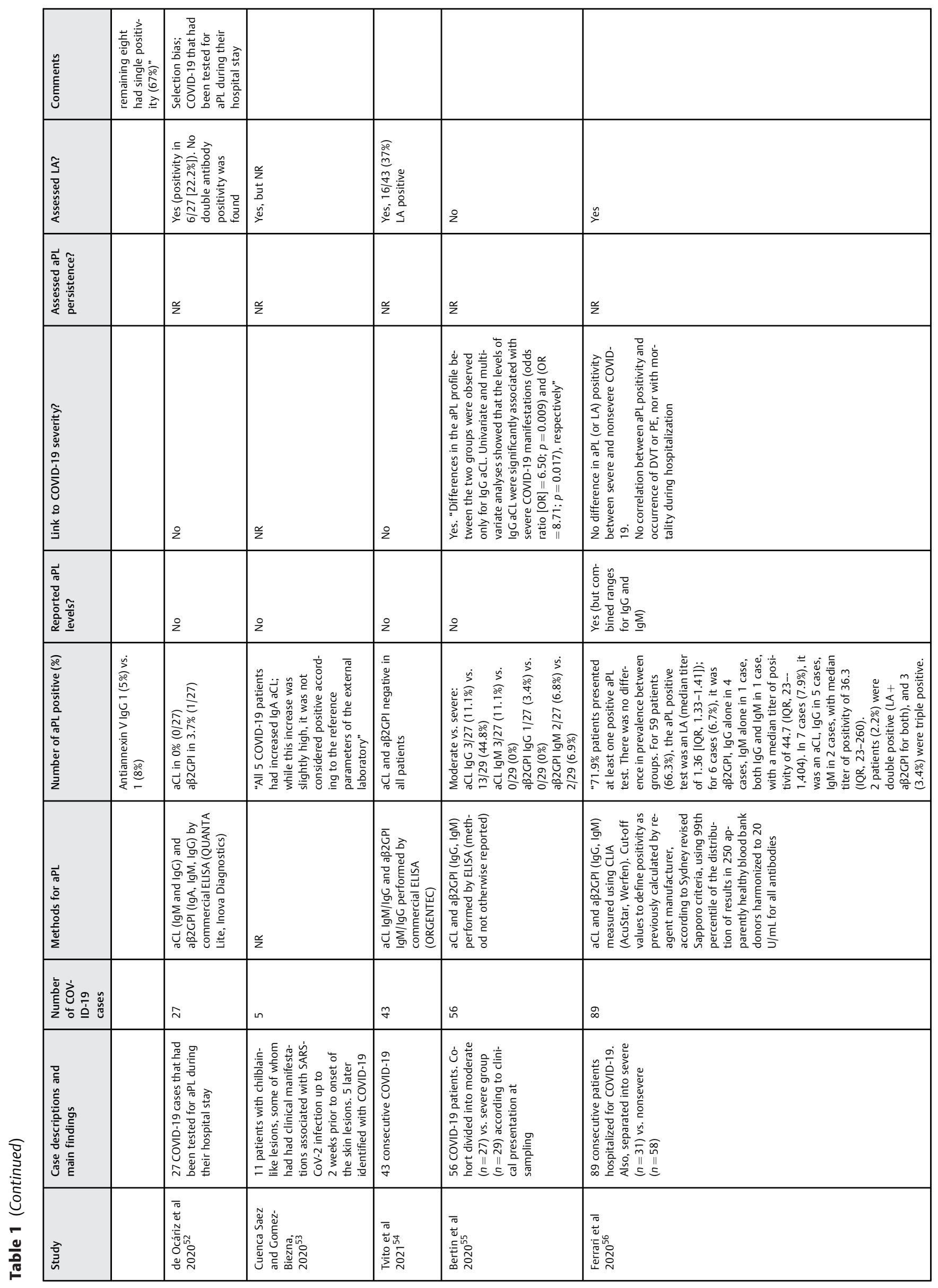

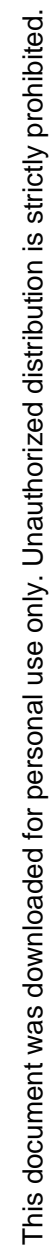




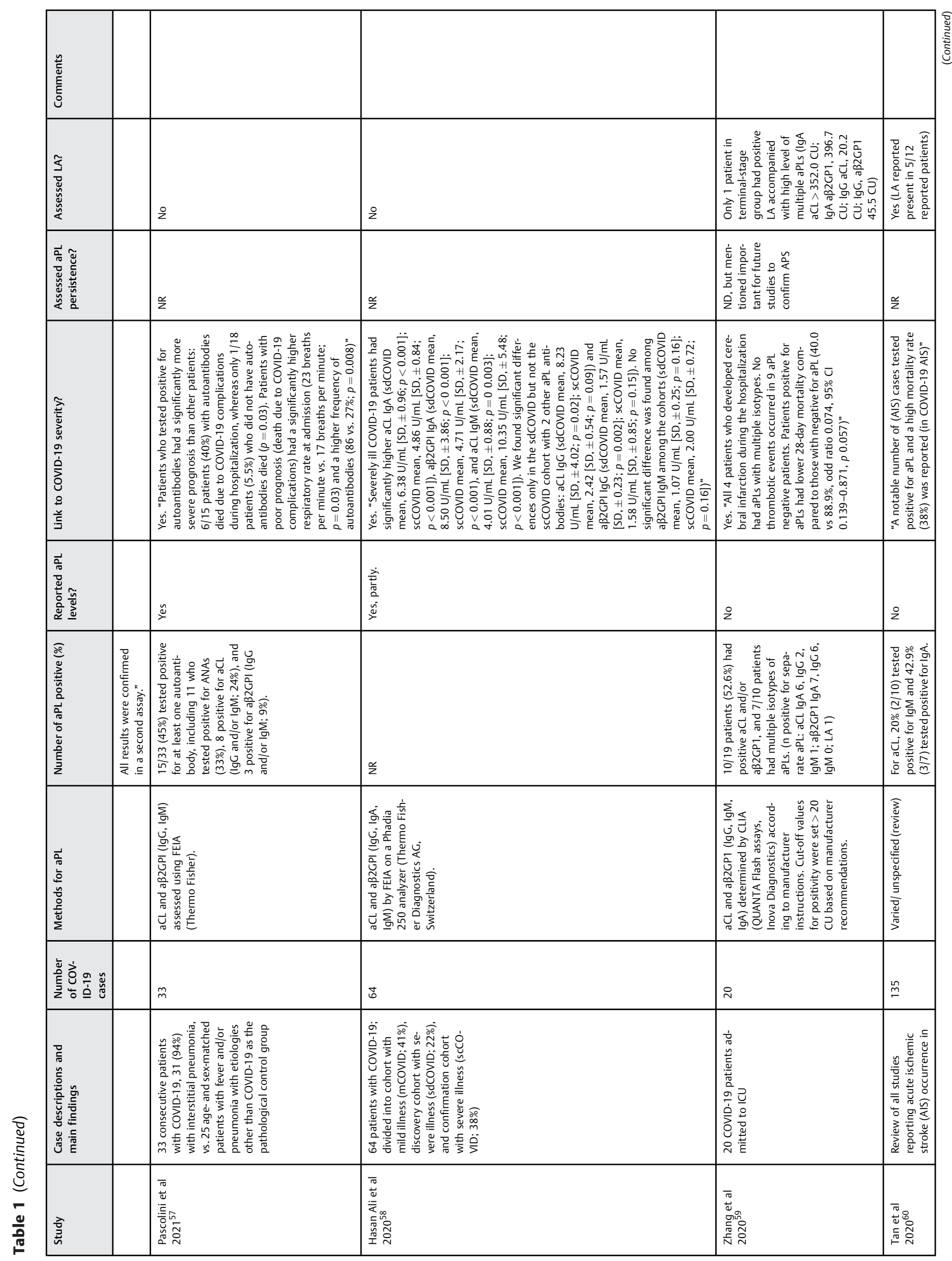




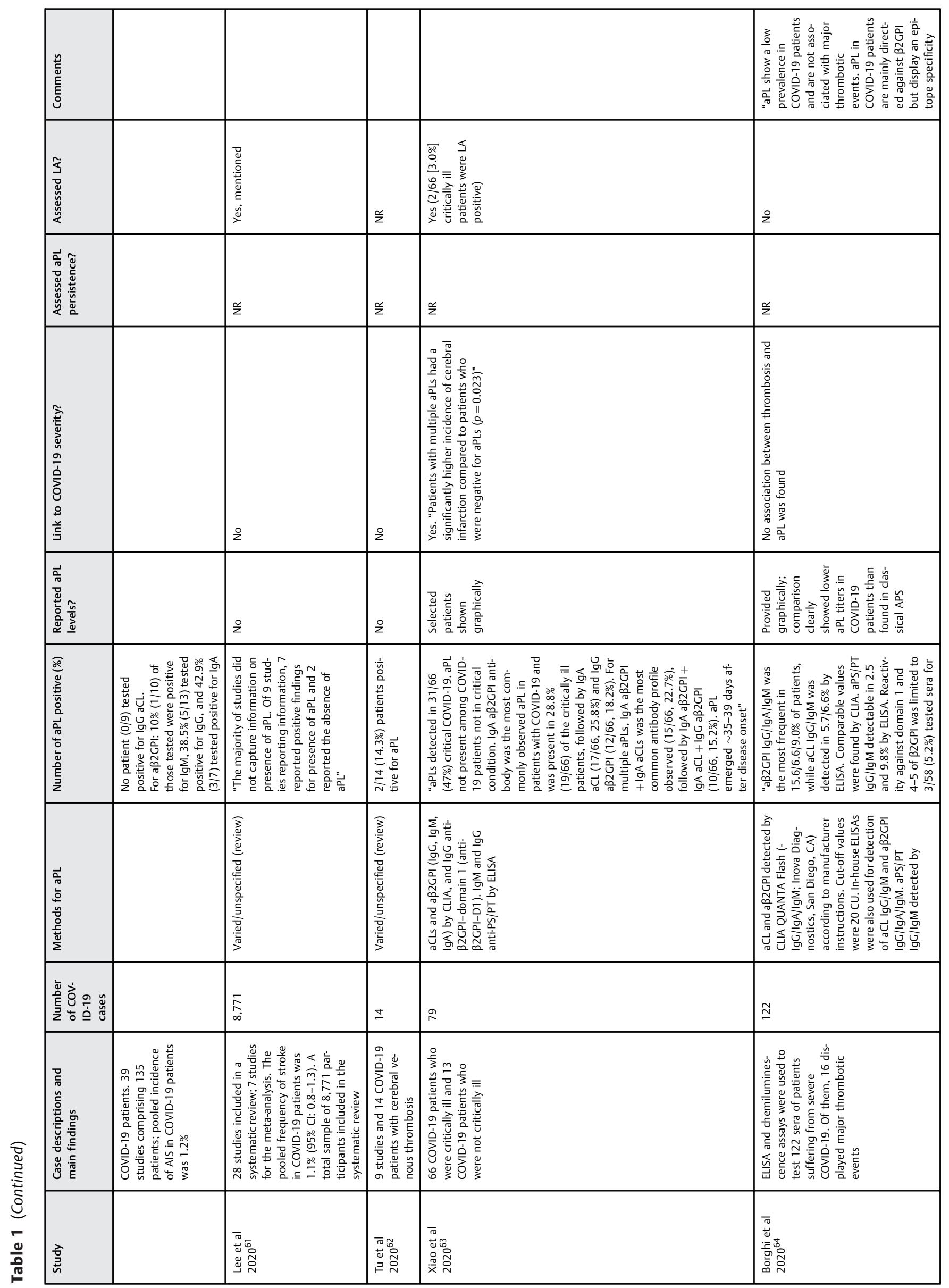


Antiphospholipid Antibodies and COVID-19 Favaloro et al.

\begin{tabular}{|c|c|c|c|c|c|c|}
\hline & 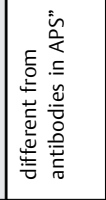 & & & 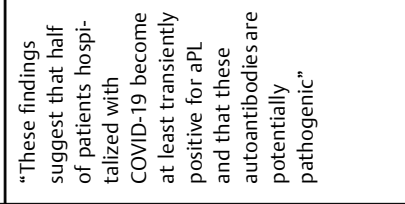 & 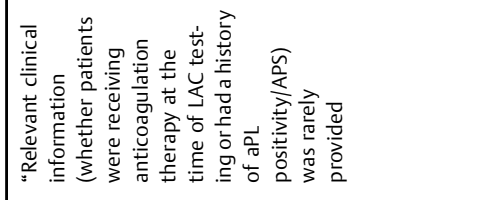 & 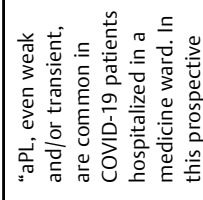 \\
\hline & & $\stackrel{\circ}{z}$ & 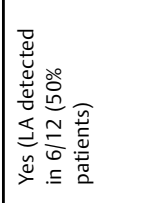 & i & $\stackrel{\check{\nu}}{\check{0}}$ & 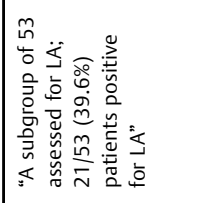 \\
\hline 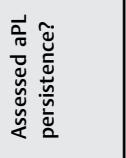 & & $\frac{o}{z}$ & $\stackrel{丷}{z}$ & 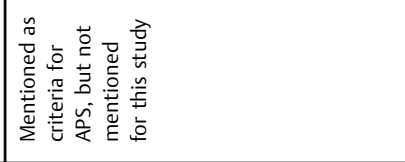 & 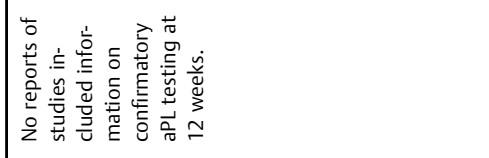 & 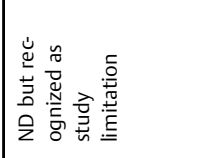 \\
\hline & &  & 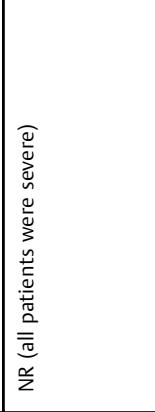 & 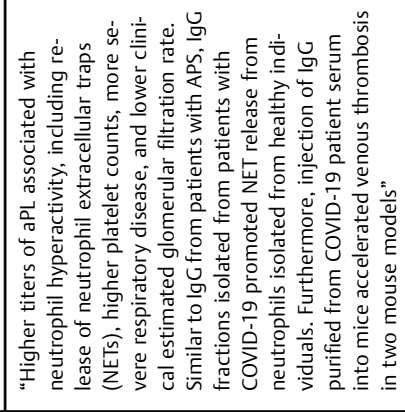 & $\stackrel{\circ}{2}$ & 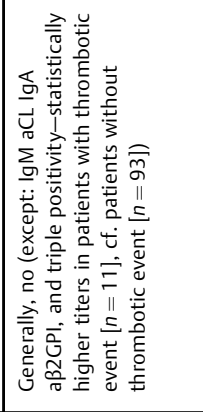 \\
\hline 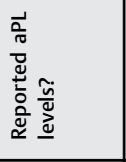 & & $\stackrel{2}{2}$ & z & z & z & 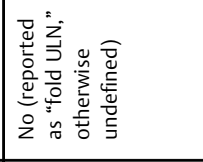 \\
\hline 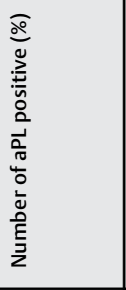 & 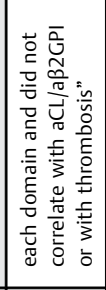 & 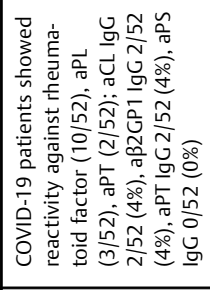 & 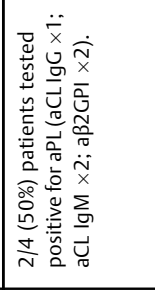 & 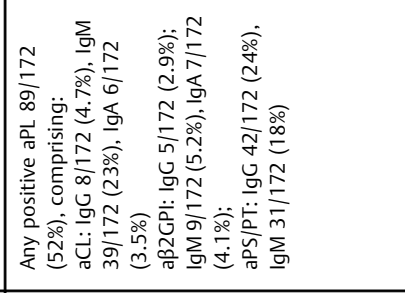 & 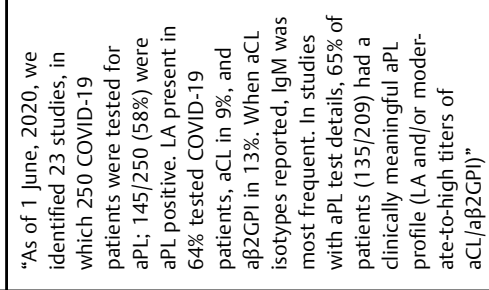 & 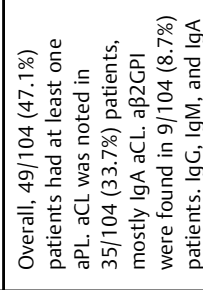 \\
\hline 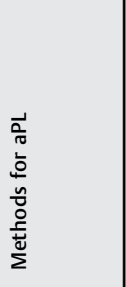 & 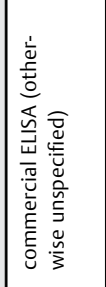 & $\frac{o}{2}$ & 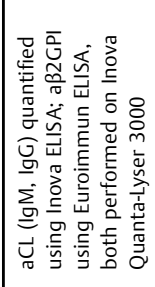 &  & 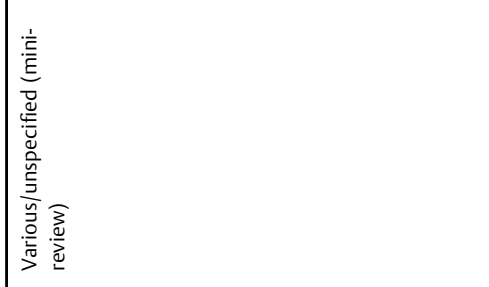 & 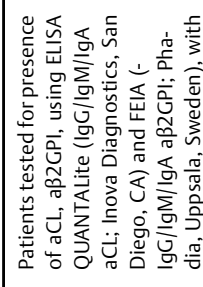 \\
\hline 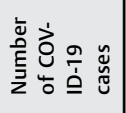 & & & $\simeq$ & $\stackrel{N}{E}$ & $\stackrel{\text { 只 }}{\text {. }}$ & $\underset{\square}{ \pm}$ \\
\hline 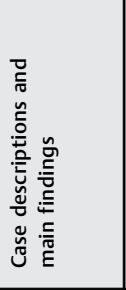 & & 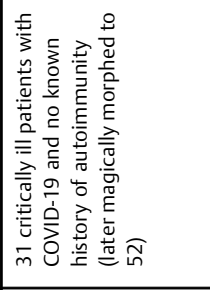 & 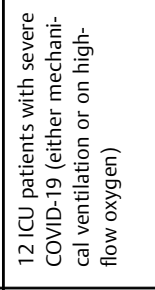 & 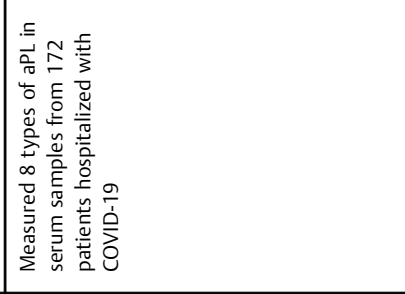 & 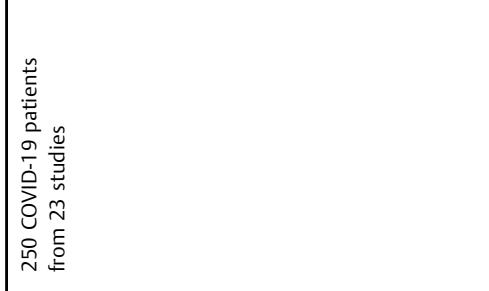 & 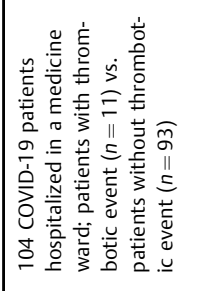 \\
\hline 总 & &  & 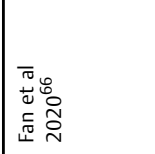 & 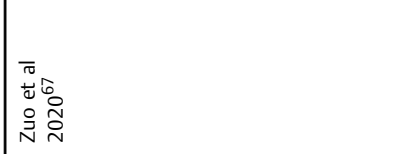 & 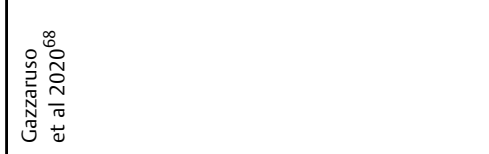 & 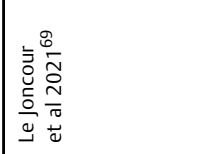 \\
\hline
\end{tabular}




\begin{tabular}{|c|c|c|c|c|}
\hline &  & 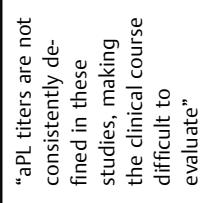 & & 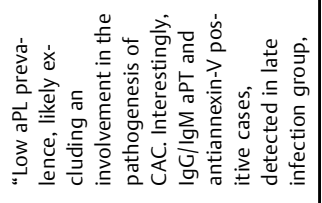 \\
\hline 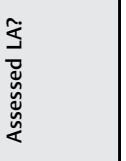 & & 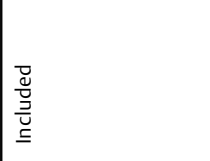 & $\stackrel{0}{z}$ & $\stackrel{0}{z}$ \\
\hline 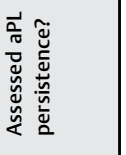 & & $\frac{\approx}{z}$ & $\frac{o}{z}$ & $\stackrel{2}{z}$ \\
\hline & & 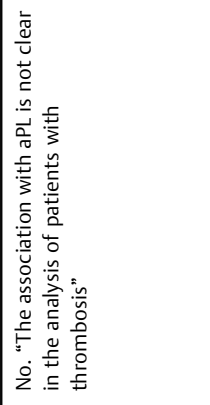 &  & 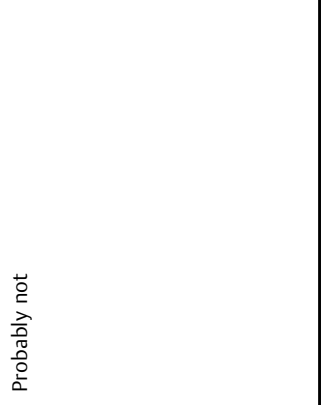 \\
\hline  & & \& & $\stackrel{2}{z}$ & 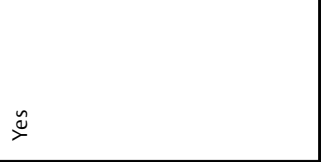 \\
\hline 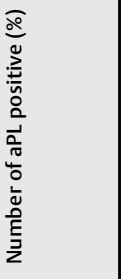 & 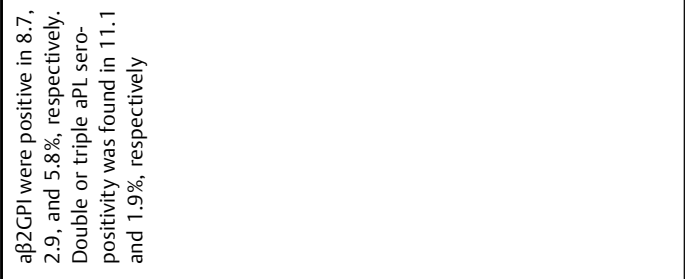 & 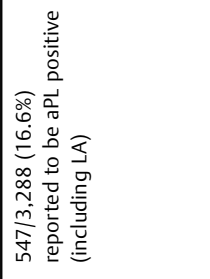 & 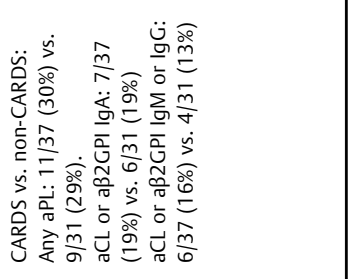 &  \\
\hline 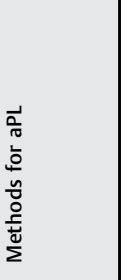 & 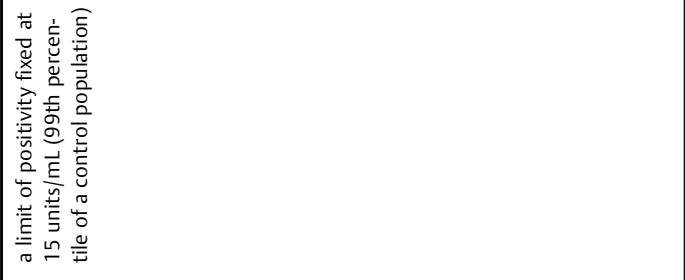 &  & $\frac{\alpha}{z}$ &  \\
\hline 总 & & 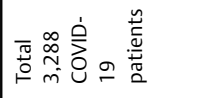 & $\hat{m}$ & $\tilde{\sigma}$ \\
\hline  & & 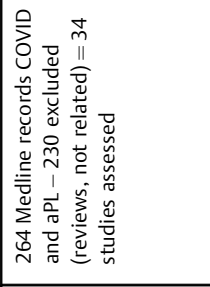 & 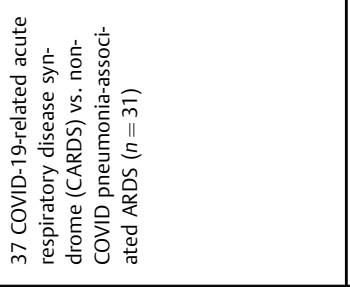 & 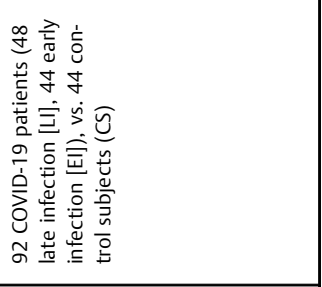 \\
\hline 总 & & \begin{tabular}{|l}
$\bar{\sigma}$ \\
$\bar{\sigma}$ \\
$\overline{\bar{v}}$
\end{tabular} &  &  \\
\hline
\end{tabular}




\begin{tabular}{|c|c|c|c|}
\hline  & 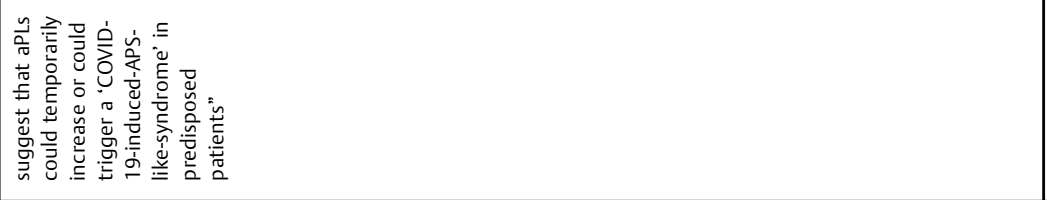 & 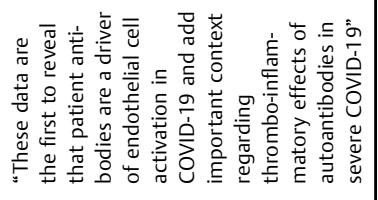 & 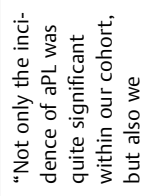 \\
\hline & & $\bar{z}$ & $\stackrel{y}{x}$ \\
\hline 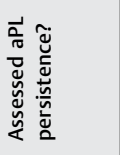 & & $\underline{z}$ & 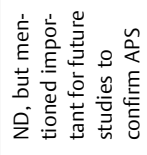 \\
\hline & &  & 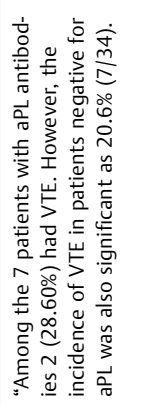 \\
\hline 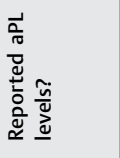 & & $\bar{z}$ & $\stackrel{\Xi}{\supset}$ \\
\hline 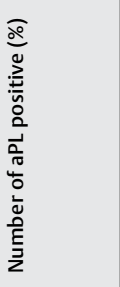 & 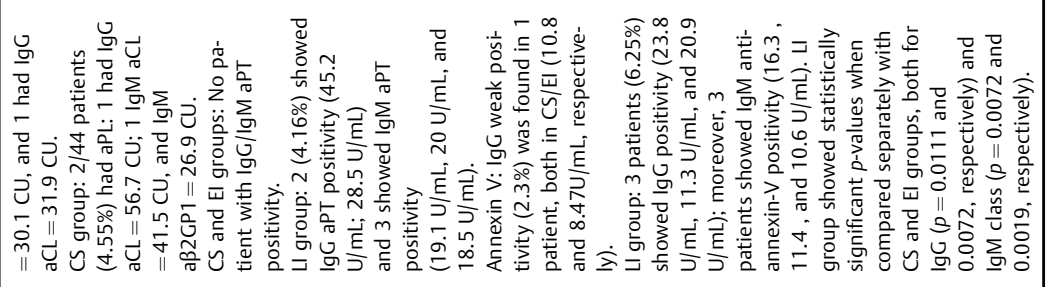 & 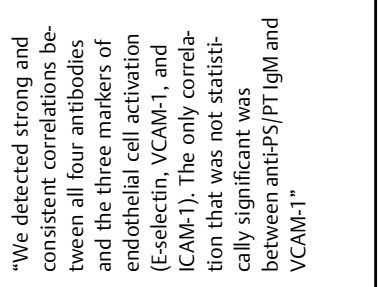 & 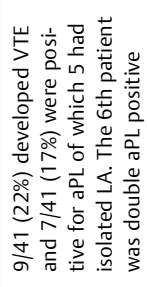 \\
\hline 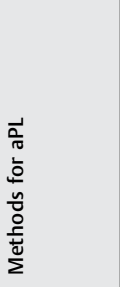 & 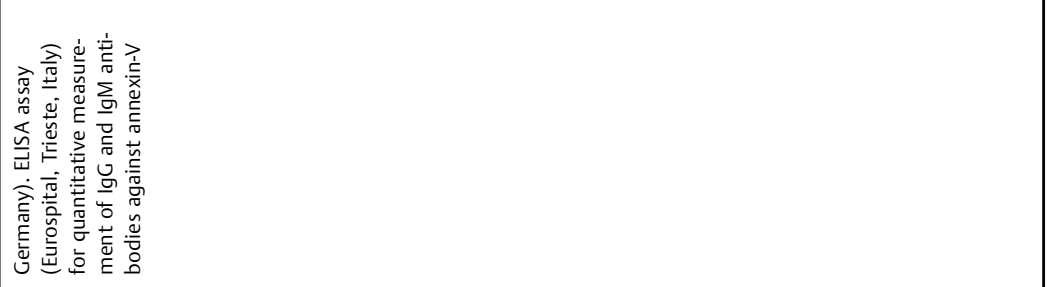 & 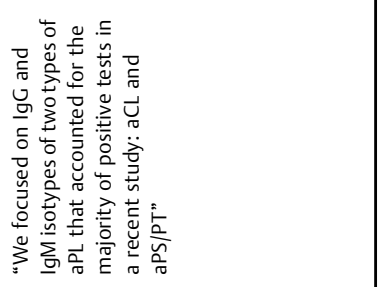 & 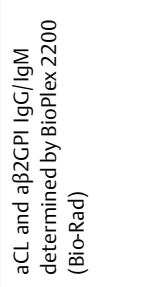 \\
\hline 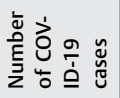 & & $\stackrel{\widehat{\infty}}{\Xi}$ & F \\
\hline 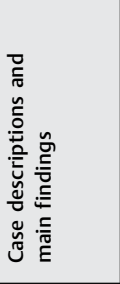 & & 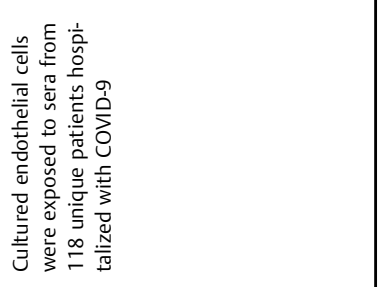 & 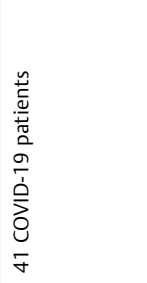 \\
\hline 离 & & 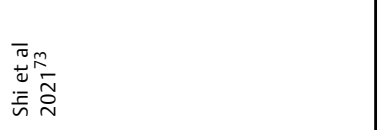 & 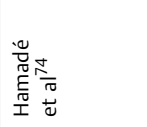 \\
\hline
\end{tabular}


84 Antiphospholipid Antibodies and COVID-19 Favaloro et al.

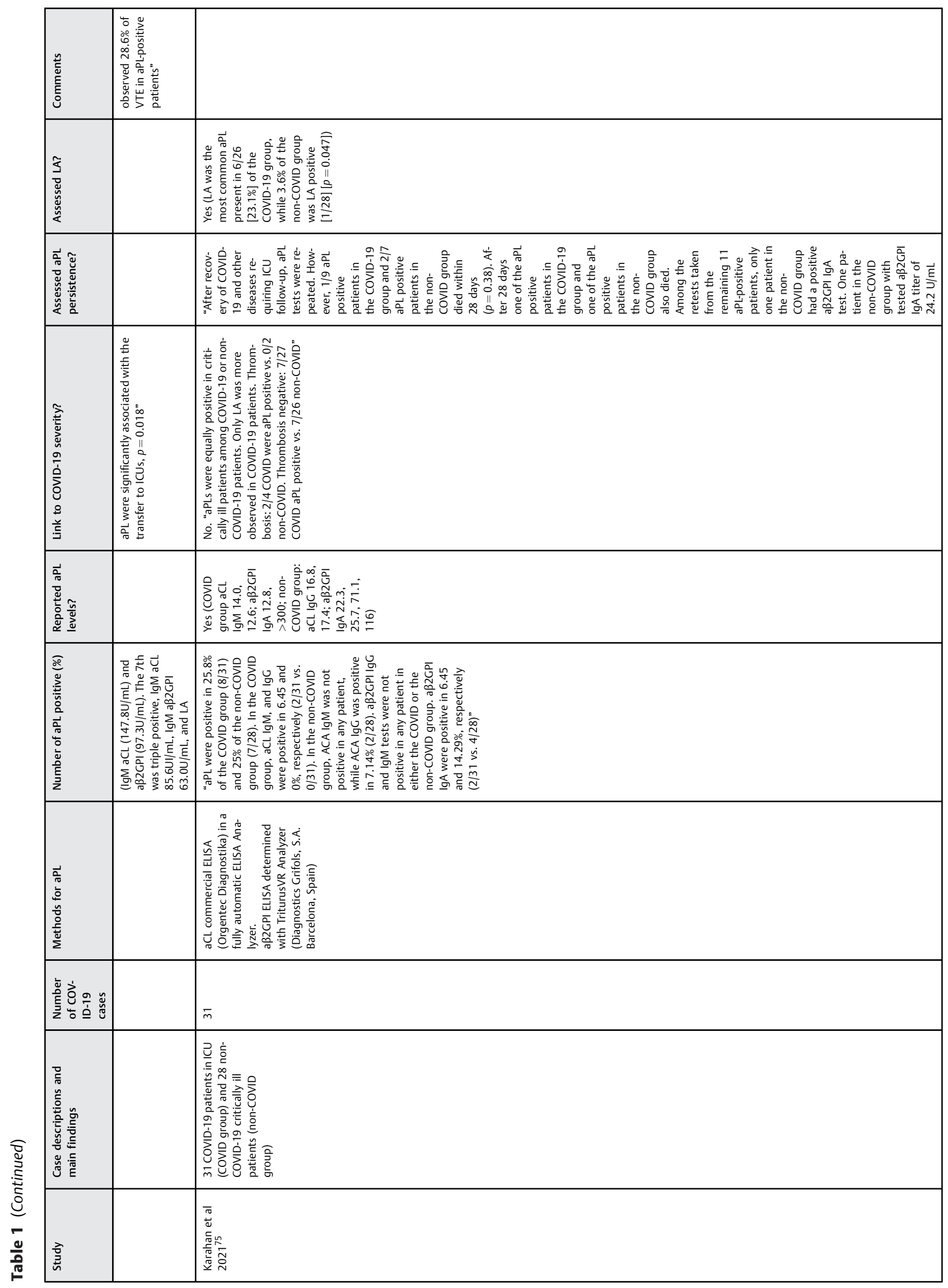




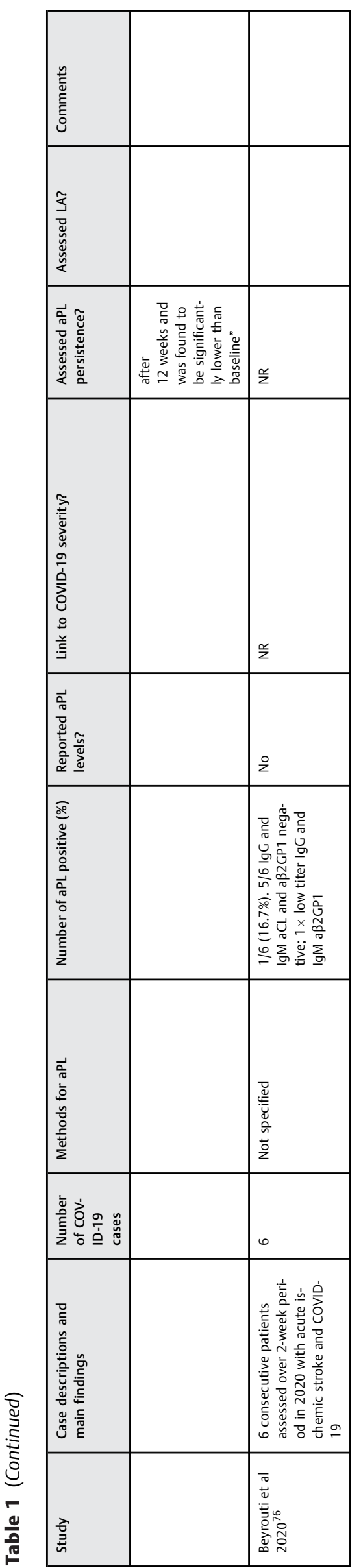

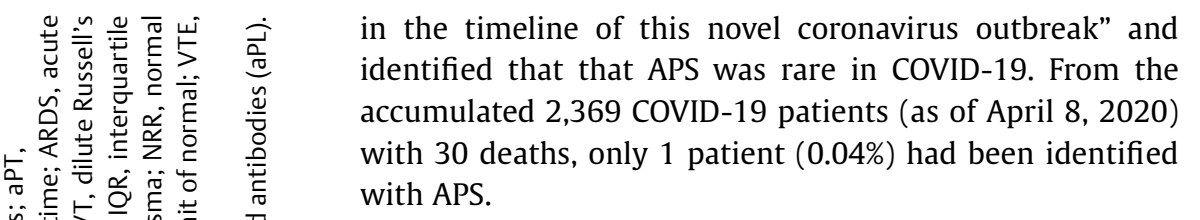

\section{Selection Bias in the Literature}

One could hypothesize that the reported incidence of COVID19-associated aPL would be higher in small cases series due to potential selection bias, as identified previously for the Zhang et al report for $\mathrm{aPL},{ }^{32}$ and as proposed also for the previous LA review. ${ }^{11}$ Thus, selection bias in the literature is likely where authors investigate aPL. First, researchers are more likely to publish positive rather than negative findings; this is particularly likely for small case series or single-case studies. Second, researchers may actively look for aPL in select COVID-19 patient cohorts, such as those with raised activated partial thromboplastin time, prompting a search for LA, or with clinical suspicion of aPL. In such studies, a relatively high incidence of aPL may be identified. One can propose that this might be anticipated, irrespective of the presence of COVID-19.

Of note, we excluded single-case studies from our literature review since these amalgamate several avenues for selection bias, and hence are more likely to publish positive case findings, and also patient selection bias. The literature on aPL also includes studies with and without investigation of LA, so aPL percentage data would fluctuate, being generally higher when LA is included..$^{11}$ Our literature search also uncovered several previous reviews on the topic of aPL in COVID-19. One review, by Novelli et al, ${ }^{70}$ identified 264 Medline records on COVID and aPL. After excluding 230 references (reviews, not related), they included 34 studies totaling 3,288 COVID-19 patients, and identified 547/3,288 (16.6\%) cases reported to be aPL positive (including LA). This review included single-case studies. For interest, we have plotted the proportion of cases positive for aPL, as reported in this review, against the number of cases included in each study in $\boldsymbol{- F i g . ~ 2 . ~ U n l i k e ~ o u r ~ r e v i e w ~ f i n d i n g s ~ ( ~}-$ Fig. 1), where no relationship was found, the data from Novelli et $\mathrm{al}^{70}$ instead show a clear statistically significant relationship, driven mostly by inclusion of many case reports of aPL positivity in COVID-19 (i.e., obviously, $100 \%$ of cases).

\section{Incidence of aPL in COVID-19}

Excluding single-case studies, the incidence of reported cases positive for aPL, in some studies also including LA, as derived from our own review, and as summarized in - Table $\mathbf{1}$ and - Fig. 1, is 33\% (median), with an interquartile range (IQR) of 11 to $52 \%$. As noted, most of the higher incidence group seem to be driven by the presence of LA, which we previously reported, ${ }^{11}$ and which was sometimes reported in $>80 \%$ of tested cases. The reported incidence of "solid phase" identified aPL (i.e., aCL, aß2GPI, etc.) was generally lower (-Table 1 and - Fig. 3). Also, of interest, a curvilinear relationship appears to exist between the 


\section{Number of COVID cases vs \% aPL positive (current review)}

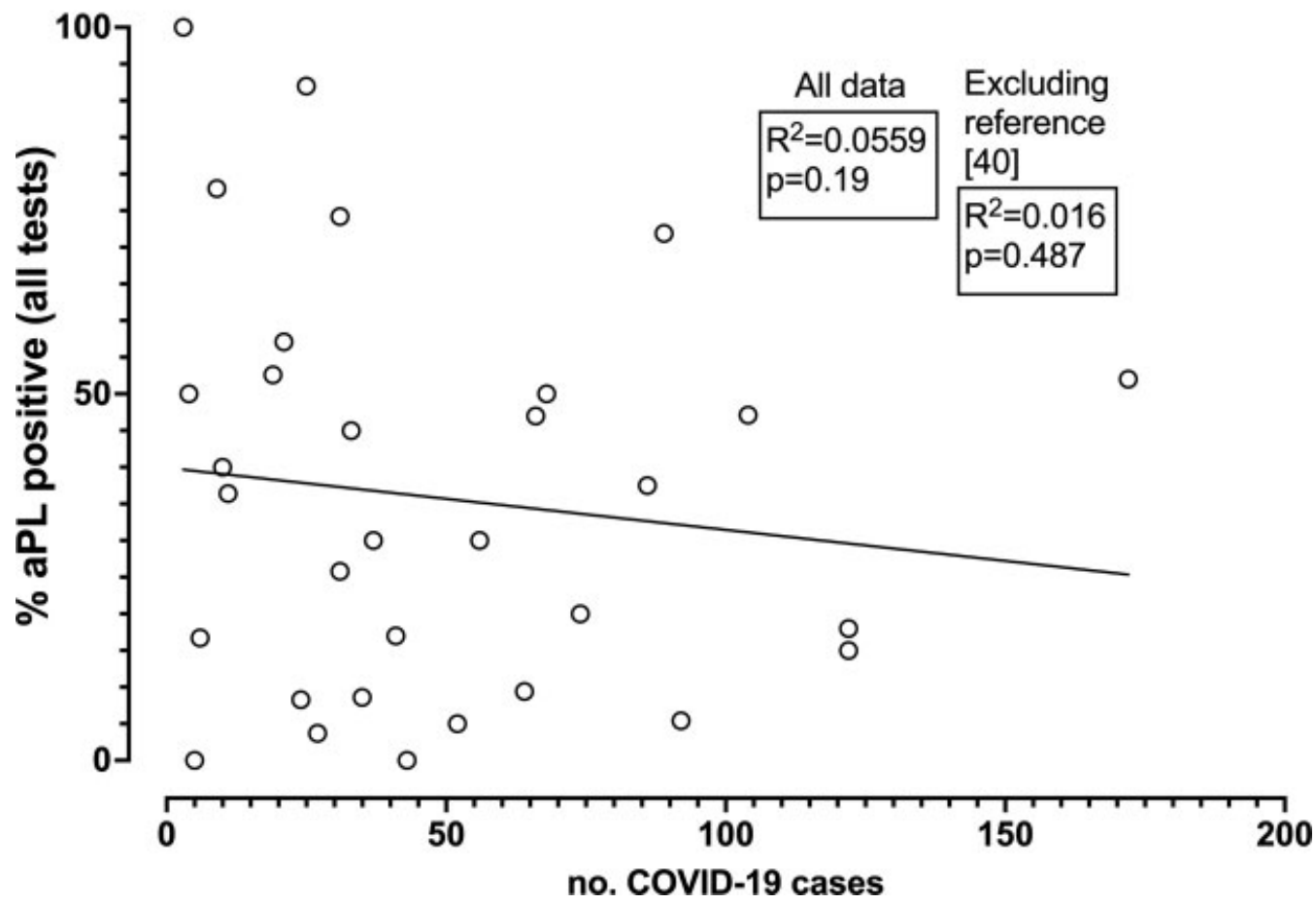

Fig. 1 The relationship (or lack thereof) between COVID-19 case numbers reported in the literature and the proportional identification of antiphospholipid antibody (aPL) positive cases, as summarized from - Table 1. The associated $R^{2}$ and $p$-value have been provided using all data from publications in - Table 1 as well as excluding the data from Yasri and Wiwanitkit. ${ }^{40}$ Where specific information on overall \% aPL positive is not given, an approximate value calculated from the presented data is included.

number of included COVID-19 cases and the incidence of some specific classes of aPL ( - Fig. 3A, B), again potentially suggestive of selection bias.

\section{Titer of aPL in COVID-19}

In general, most aPL, where identified, were of fairly low titer, and thus high-titer aPL were rarely identified (-Table 1).

\section{Criteria aPL (Excluding LA)}

-Fig. 4 provides a summary of aPL titers reported in the literature for APS "criteria" aPL (but excluding LA, as reported elsewhere ${ }^{11}$ ). In some cases, the range of aPL data was provided for the entire COVID-19 cohort, and in some other cases, it was only reported for patients positive for aPL. The perception of titer for COVID-19 obviously increases when only positive cases are considered. Two studies provided numerical data for a series of reported cases, allowing this differential to be highlighted. Thus, individual values for a reasonable number of COVID-19 cases were provided by Devreese et $\mathrm{al}^{49}$ and Pineton de Chambrun et ${ }^{4},{ }^{46}$ permitting calculation of median and IQR values both for their entire aPL cohorts and for only positive cases (-Fig. 4).

Excluding LA, which was the subject of the prior review, ${ }^{11}$ aCL titers for COVID-19 cohorts were often in the same range as the expected "normal reference range," which generally used a cut-off of 20 units for both $\operatorname{IgG}$ and IgM classes, noting here that different methods may use different measurement units (e.g., GPL units for some ELISA-based assays, in line with the original "Harris" standards, vs. CU for the CLIA method on AcuStar/BioFlash). Naturally, considering only positive aPL cases, the aPL titer by definition will exceed the cut-off value, but even here could only be considered as a low titer in most studies (e.g., $<40$ units). Although ranges on occasion did include high titers in some studies, these were in the minority and for only a few patients overall. In two studies, a comparison was made with COVID-19 and "classical" APS cohorts. ${ }^{43,64}$ Data from one of these studies, from Gatto et $\mathrm{al}^{43}$ are included in - Fig. 4. These authors studied a relatively large number of cases $(n=122)$, divided into hospitalized and nonhospitalized COVID-19 cases, as well as a separate control group of "other autoimmune rheumatic diseases." Borghi et al ${ }^{64}$ also studied 122 COVID-19 patients and provided comparative data with an APS cohort, represented graphically in their report. In both studies, ${ }^{43,64}$ the titers identified in data from their APS cohorts greatly exceeded those identified in the COVID-19 cohorts.

As per general studies on aPL, the most common criteria aPL identified in COVID-19 studies, and those tending to include higher titer aPL cases, were for aCL IgG and IgM and aß2GPIb IgG. aß2GPIb IgM positivity or high titer was less often identified (-Table 1, - Fig. 3A, and - Fig. 4).

\section{Noncriteria aPL}

A large number of noncriteria aPL have also been investigated in COVID-19 (-Table 1). Among these studies, most data are available for aCL IgA and aß2GPIb IgA, and then for aPS/aPT. 
A. Number of COVID cases vs $\%$ aPL positive from ref [70]; all data

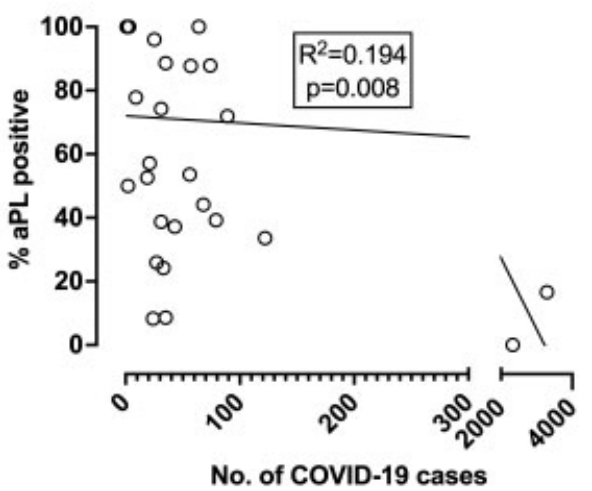

B. Number of COVID cases vs \% aPL positive from ref [70]; excluding ref [40]

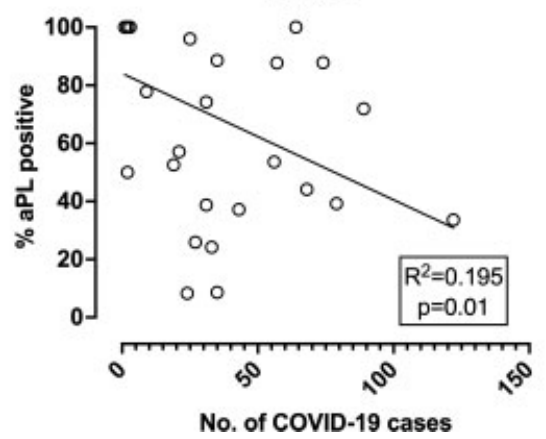

Fig. 2 The relationship between COVID-19 case numbers reported in the literature and the proportional identification of antiphospholipid antibody (aPL) positive cases, using data from Novelli et al. ${ }^{70}$ (A) Data including those from Yasri and Wiwanitkit ${ }^{40}$ who reported a single case of APS, presumably having positive aPL, from 2,369 COVID-19 patients. (B) Data excluding that from Yasri and Wiwanitkit. ${ }^{40}$

Some data on titer are also provided (-Table 1, - Fig. 5). In general, fewer cases of COVID-19 were found with noncriteria aPL than criteria aPL ( - Table 1 and - Fig. 3B, C), and where reported, titers tended to be similar to those of criteria aPL (-Table 1 and -Fig. 5), except for occasional patients.

\section{Multiple Positivity for aPL}

Multiple positivity for aPL was rarely reported. Thus, double and triple positivity was only reported for a few isolated individuals (- Table 1). ${ }^{49,51,56,59,63,69,74}$

\section{Persistence of aPL Positivity versus Transient Positivity}

To identify aPL as a specific feature of an autoimmune disorder such as APS, one has to prove the persistence of that positivity, generally by repeating the test(s) on a second sample some 12 weeks after the first positive test result. ${ }^{1,2,37-39}$ Again, most researchers reporting on LA positivity in COVID-19 either did not mention this or did not undertake repeated testing. Thus, persistence of aPL positivity was not evaluated in most studies, and hence could not be proven. In the two studies that did attempt to look at
A.

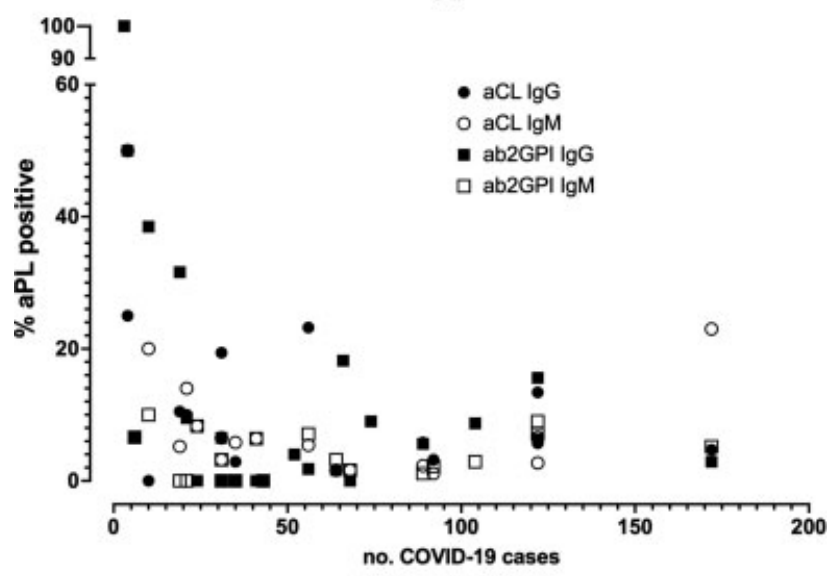

B.

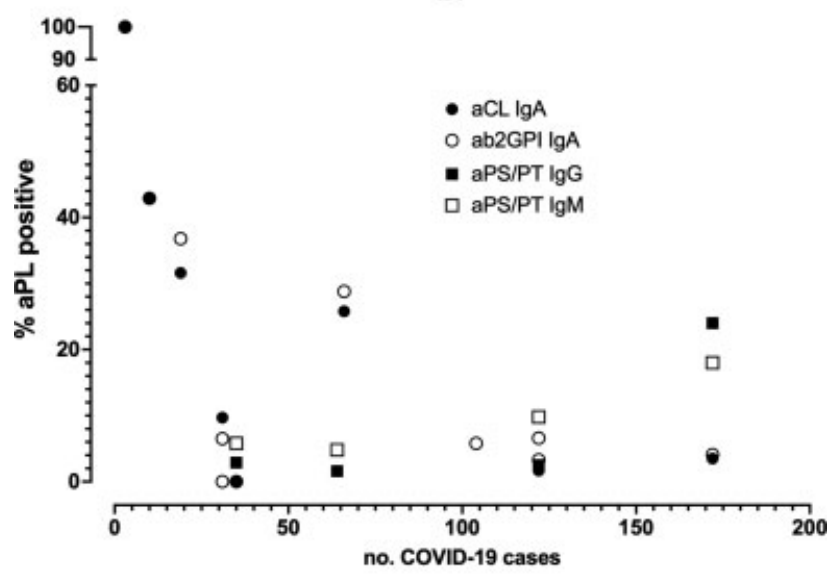

C.

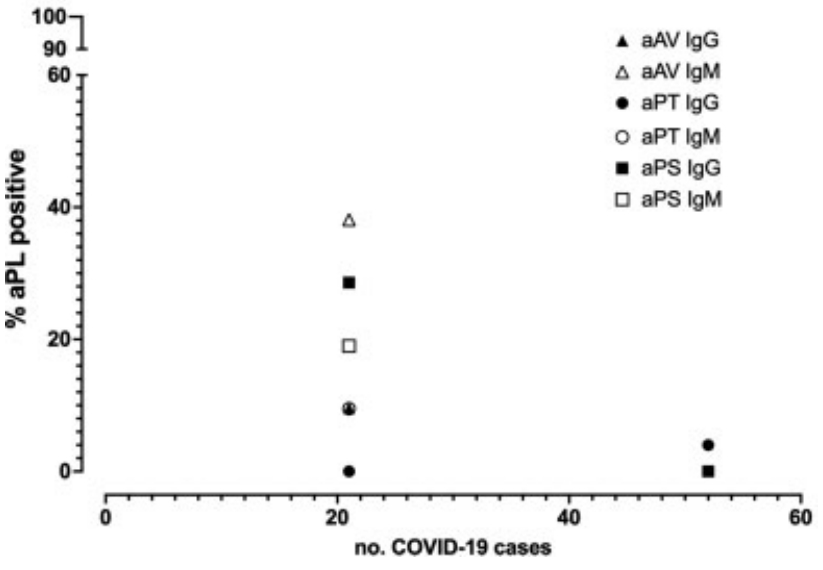

Fig. 3 The relationship between COVID-19 case numbers reported in the literature and the proportional identification of solid-phasedetected antiphospholipid antibody (aPL) positive cases according to tested aPL. (A) "Criteria" aPL and (B, C) "noncriteria" aPL. aAV, antiannexin $\mathrm{V}$ antibodies; $\mathrm{aCL}$, anticardiolipin antibodies; $\mathrm{a} \beta 2 \mathrm{CPI}$, anti- $\beta 2$-glycoprotein I antibodies; aPS, antiphosphatidylserine antibodies; aPT, antiprothrombin antibodies; aPS/PT,

antiphosphatidylserine/prothrombin complex antibodies.

persistence, most cases initially positive for aPL then became negative for $\mathrm{aPL},{ }^{49}$ or else repeat testing was complicated by the ongoing patient morbidity or their death. ${ }^{75}$ Thus, it 

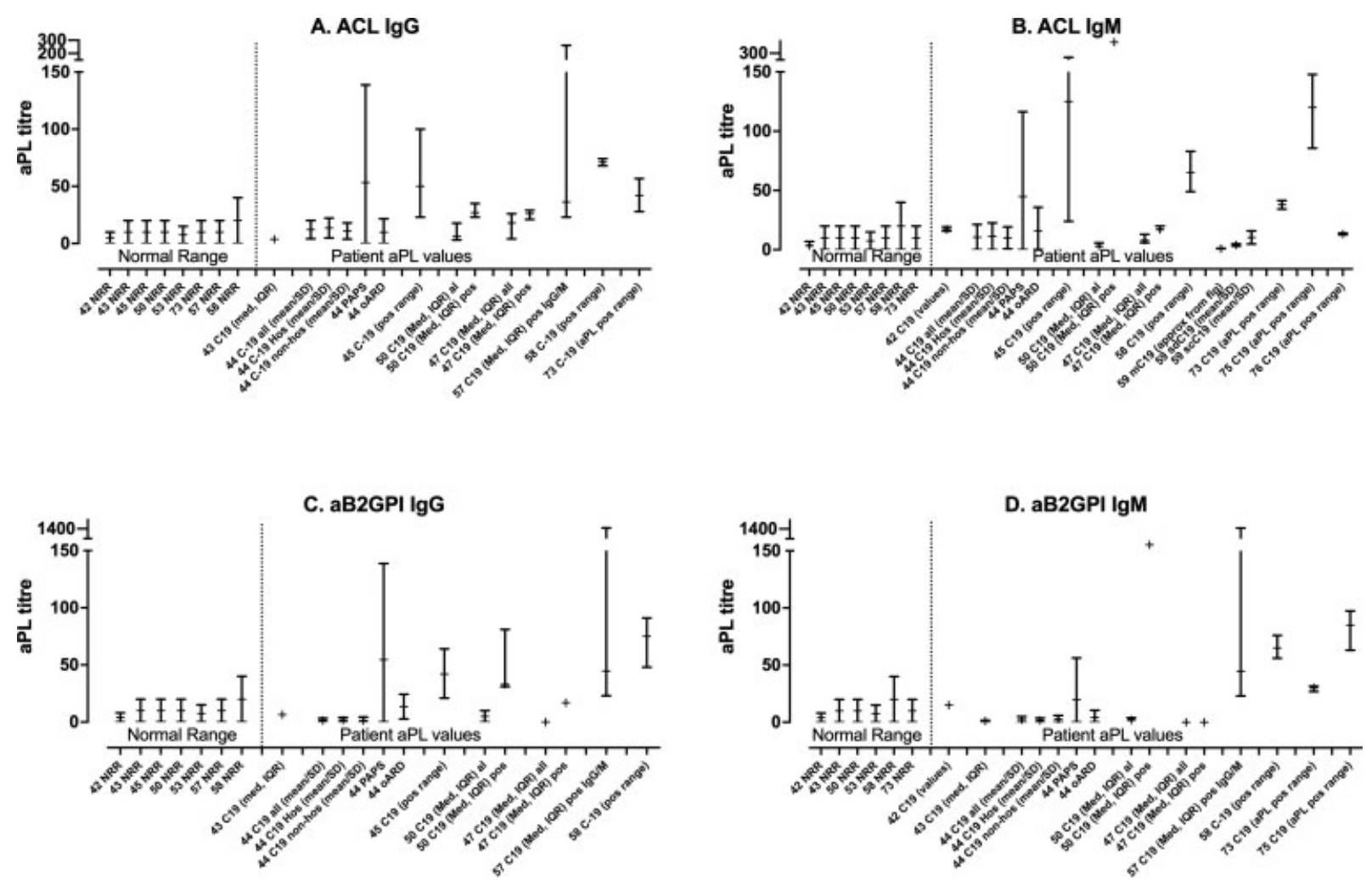

Fig. 4 (A-D) Summary findings for antiphospholipid (antibody) syndrome (APS) "criteria" antiphospholipid antibodies (aPL) (excluding LA) from - Table 1 showing the range of normal expected findings (NRR, normal reference range) vs. cases of COVID-19. Note that the study reference number is provided in the text detail along the $\mathrm{x}$-axis. The ranges reported in the literature reflect different data capture methods, including mean \pm standard deviation (SD), or median/interquartile range (IQR), or individual values. Sometimes ranges are for entire COVID-19 cohorts (i.e., including aPL-negative cases) and sometimes only for aPL-positive cases. Naturally, by definition, where only positive aPL case values are provided, these will inevitably be higher than those of NRRs or entire cohort values. In cases where titer value ranges included high titers, these were invariably small case numbers out of the entire COVID-19 cohort. aCL, anticardiolipin antibodies; a 32 CPI, anti- $\beta 2$-glycoprotein I antibodies.

seems that any aPL positivity that may be identified in COVID-19 patients is mostly transient.

\section{Transient aPL Are a Common Feature of Severe Viral Infections}

As extensively discussed in the companion review on LA, ${ }^{11}$ sick patients with various viral infections in a range of conditions may have aPL appear transiently. ${ }^{77,78}$ It may also be possible to separate groups of patients and aPL profiles. Abdel-Wahab et $\mathrm{al}^{77}$ for example, reported three different groups of patients following viral infection; "group 1 included patients who fulfilled the criteria for definitive APS (24.6\%), group 2 included patients who developed transient aPL with thromboembolic phenomena (43.7\%), and group 3 included patients who developed transient aPL without thromboembolic events (31.7\%)." Thus, secondary cases of APS due to viral infections have been reported. ${ }^{78}$ Secondary cases of APS due to infectious agents potentially evolving into CAPS have also been reported and include infections from hepatitis C virus, herpes zoster, bacteria, fungi, and parasites and acute $\mathrm{Q}$ fever. ${ }^{79}$ The induction of molecular mimicry that leads to production of a $32 \mathrm{GPI}$ autoantibodies has been proposed as putative cause of secondary APS and CAPS. ${ }^{80,81}$
Thus, the finding of aPL positivity in COVID-19 is not unique to COVID-19. To our knowledge, there is no evidence available on comparative infections with other viral agents to identify if the situation in COVID-19 with respect to aPL positivity is worse or greater than that of other severe viral infections. In part, it is also likely that other viral diseases have not been as extensively studied as COVID-19, owing to their relatively lower epidemiologic burden.

\section{Does aPL Positivity in COVID-19 Reflect a Risk Factor for Thrombosis?}

Only a few studies investigated whether aPL positivity inferred additional thrombotic risk. Few studies identified a statistical difference in thrombotic risk for aPL-positive versus aPL-negative patients or substantial aPL positivity in their thrombosis cohorts (LA only ${ }^{45,50}$ ), $55,57-59,63$ whereas most did not. $31,41-44,47-49,52,54,56,61,62,64,69-72,74,75$ There are many potential confounders to consider, and it is unlikely that such confounders were considered in all published comparisons. Thus, transient aPL positivity may develop in the sickest patients, who will then be most at risk of thrombosis, and therefore aPL may just reflect an association with, rather than be responsible for, the pathophysiological events. Irrespective, whether aPL positivity in COVID-19 

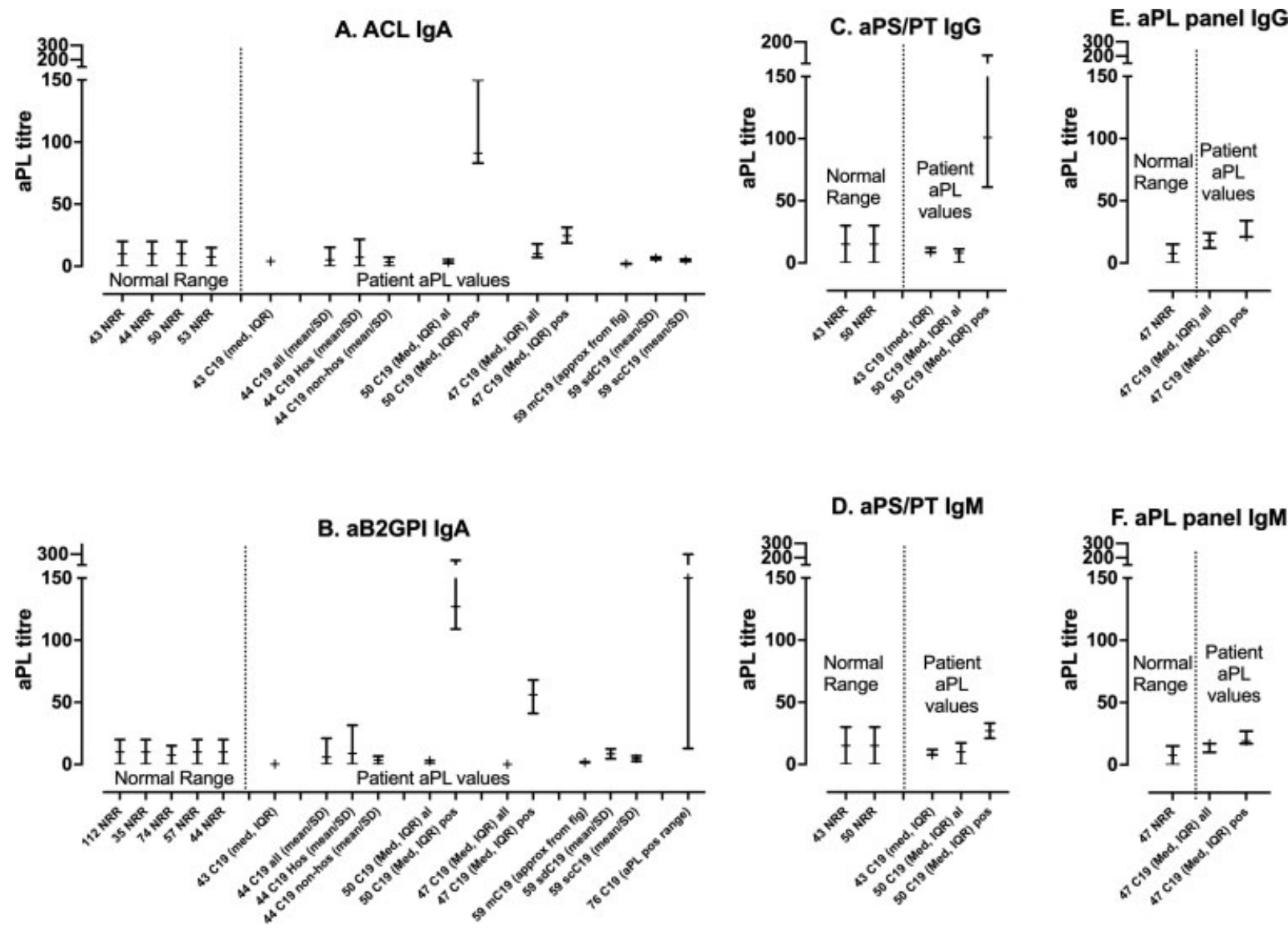

Fig. 5 (A-F) Summary findings for antiphospholipid (antibody) syndrome (APS) "noncriteria" antiphospholipid antibodies (aPL) from - Table 1 showing the range of normal expected findings (NRR, normal reference range) vs. cases of COVID-19. Note that the study reference number is provided in the text detail along the $x$-axis. The ranges reported in the literature reflect different data capture methods, including mean \pm standard deviation (SD), or median/interquartile range (IQR), or individual values. Sometimes ranges are for entire COVID-19 cohorts (i.e., including aPL-negative cases) and sometimes only for aPL positive cases. Naturally, by definition, where only positive aPL case values are provided, these will inevitably be higher than those of NRRs or entire cohort values. In cases where titer value ranges included high titers, these were invariably small case numbers out of the entire COVID-19 cohort. aCL, anticardiolipin antibodies; a $22 \mathrm{GPI}$, anti- $\beta 2$-glycoprotein I antibodies; aPS/PT, antiphosphatidylserine/prothrombin complex antibodies.

truly reflects an additional risk factor for thrombosis remains currently unresolved. Consistent with patterns identified in APS, ${ }^{1,2}$ those with multiple aPL positivity in COVID-19 are most likely to have an association with thrombosis, and double and triple positivity was only identified in a few patients in a few publications. $49,51,56,59,63,69,74$

\section{Conclusion}

Taking all this information into consideration, we need to recognize that aPL positivity does represent a feature of COVID-19, at least in some patients, and potentially those who are the sickest or have the most severe infection. However, this does not indicate APS or CAPS for most patients. Also, there appears to be a higher proportion of LA identified in patient cohorts ${ }^{11}$ than "solid-phase" aPL, as identified in this review, although additional confounders exist in LA identification in COVID-19. ${ }^{11}$

Nevertheless, additional confounders also need to be considered for other aPL. In particular, repeat testing for persistence of aPL was rarely performed or described, and when reported, suggested a transient nature of the identified
aPL. Such transient aPL do not identify an autoimmune disease in the classic sense of APS, ${ }^{1,2}$ can be commonly observed in other viral infections, ${ }^{77,78}$ and thus do not seem to be unique to COVID-19. There are also questions remaining over the "additional" thrombotic risk imposed by the aPL identified in COVID-19 in these studies, as transient aPL developed from viral infections are often not associated with thrombosis. In regard to incidence and titer, not only was the typical incidence of "solid-phase" aPL often low, but also, where identified, the titer was also generally low. This was true of both criteria "solid-phase" aPL (aCL and aß2GPI of IgG and IgM class; - Fig. 4) and noncriteria aPL (-Fig. 5). Certainly, where comparative data for "classical" APS were given, ${ }^{49,64}$ the titers found in COVID-19 patients were considerably lower. In those case where high proportions of "solid-phase" aPL were detected, the cut-off value used to define positivity was not always identified; if a low cut-off is applied, this will then identify a higher number of positive cases. In any case, titers considered medium or high (i.e., generally $>40$ ) were in the minority, as were those with multiple positivity, both of which increase the likely association with thrombosis. 
Thus, better studies are needed to address the residual question regarding the true frequency of aPL in COVID-19, and whether these laboratory-detected aPL truly contribute to enhance the thrombotic risk in COVID-19. Nevertheless, some good-quality studies have already been published, and these should likely guide opinion. These studies are those that reported on aPL cognizant of the potential confounders, including C-reactive protein and anticoagulant therapy for LA, and that also looked at persistence of antibodies, titer of aPL, and multiple positivity, and provide comparative data with classical APS. However, these were in the minority of published studies. All this is not to say that APS cannot develop in patients with COVID-19. As already mentioned, there are certainly similarities between the worst presentation of APS, namely, CAPS, and what occurs in the sickest patients with COVID-19. However, there are also some notable differences, including general lack of high-titer aPL, lack of persistence for aPL, and unclear relationship between the detected aPL and COVID-19-associated coagulopathy, along with many other prothrombotic abnormalities (e.g., endothelial cell injury, platelet hyperactivation, prolonged immobilization) that would justify an enhanced thrombotic risk by themselves. Also, some patients with COVID-19 must by chance have APS, and thus these may reflect a coincident finding.

\section{Conflict of Interest}

None declared.

\section{Acknowledgments}

The opinions expressed in this review are those of the authors, and do not necessarily reflect the opinions of their respective employers, NSW Health Pathology, The Heart Institute, Cincinnati Children's Hospital Medical Center, and the University of Verona.

\section{References}

1 Miyakis S, Lockshin MD, Atsumi T, et al. International consensus statement on an update of the classification criteria for definite antiphospholipid syndrome (APS). J Thromb Haemost 2006;4 (02):295-306

2 Devreese KMJ, Ortel TL, Pengo V, de Laat BSubcommittee on Lupus Anticoagulant/Antiphospholipid Antibodies. Laboratory criteria for antiphospholipid syndrome: communication from the SSC of the ISTH. J Thromb Haemost 2018;16(04): 809-813

3 Tkachenko O, Lapin S, Mazing A, et al. Profiling of non-criteria antiphospholipid antibodies in patients with SLE: differentiation of thrombotic SLE patients and risk of recurrence of thrombosis. Lupus 2020;29(05):490-498

4 Liu T, Gu J, Wan L, et al. "Non-criteria" antiphospholipid antibodies add value to antiphospholipid syndrome diagnoses in a large Chinese cohort. Arthritis Res Ther 2020;22(01):33

5 Žigon P, Podovšovnik A, Ambrožič A, et al. Added value of noncriteria antiphospholipid antibodies for antiphospholipid syndrome: lessons learned from year-long routine measurements. Clin Rheumatol 2019;38(02):371-378

6 Zohoury N, Bertolaccini ML, Rodriguez-Garcia JL, et al. Closing the serological gap in the antiphospholipid syndrome: the value of "non-criteria" antiphospholipid antibodies. J Rheumatol 2017;44 (11):1597-1602
7 Favaloro EJ, Wong RC. Antiphospholipid antibody testing for the antiphospholipid syndrome: a comprehensive practical review including a synopsis of challenges and recent guidelines. Pathology 2014;46(06):481-495

8 Lakos G, Favaloro EJ, Harris EN, et al. International consensus guidelines on anticardiolipin and anti- $\beta 2$-glycoprotein I testing: report from the 13th International Congress on Antiphospholipid Antibodies. Arthritis Rheum 2012;64(01):1-10

9 Pierangeli SS, de Groot PG, Dlott J, et al. 'Criteria' aPL tests: report of a task force and preconference workshop at the 13th International Congress on Antiphospholipid Antibodies, Galveston, Texas, April 2010. Lupus 2011;20(02):182-190

10 Galli M, Luciani D, Bertolini G, Barbui T. Lupus anticoagulants are stronger risk factors for thrombosis than anticardiolipin antibodies in the antiphospholipid syndrome: a systematic review of the literature. Blood 2003;101(05):1827-1832

11 Favaloro EJ, Henry BM, Lippi G. Is lupus anticoagulant a significant feature of COVID-19? A critical appraisal of the literature. Semin Thromb Hemost 2021; Doi: 10.1055/s-0041-1729856

12 COVID-19 Dashboard by the Center for Systems Science and Engineering (CSSE) at Johns Hopkins University. Accessed March 16 2021 at: https://www.arcgis.com/apps/opsdashboard/index. html\#/bda7594740fd40299423467b48e9ecf6

13 Lippi G, Sanchis-Gomar F, Favaloro EJ, Lavie CJ, Henry BM. Coronavirus disease 2019-associated coagulopathy. Mayo Clin Proc 2021;96(01):203-217

14 Di Minno A, Ambrosino P, Calcaterra I, Di Minno MND. COVID-19 and venous thromboembolism: a meta-analysis of literature studies. Semin Thromb Hemost 2020;46(07):763-771

15 Jenner WJ, Kanji R, Mirsadraee S, et al. Thrombotic complications in 2928 patients with COVID-19 treated in intensive care: a systematic review. J Thromb Thrombolysis 2021;51(03): 595-607

16 Uaprasert N, Moonla C, Sosothikul D, Rojnuckarin P, Chiasakul T. Systemic coagulopathy in hospitalized patients with coronavirus disease 2019: a systematic review and meta-analysis. Clin Appl Thromb Hemost 2021;27:1076029620987629

17 Carsana L, Sonzogni A, Nasr A, et al. Pulmonary post-mortem findings in a series of COVID-19 cases from northern Italy: a twocentre descriptive study. Lancet Infect Dis 2020;20(10):1135-1140

18 Wichmann D, Sperhake JP, Lütgehetmann M, et al. Autopsy findings and venous thromboembolism in patients with COVID-19: a prospective cohort study. Ann Intern Med 2020;173(04):268-277

19 Bradley BT, Maioli H, Johnston R, et al. Histopathology and ultrastructural findings of fatal COVID-19 infections in Washington State: a case series. Lancet 2020;396(10247):320-332

20 Varga Z, Flammer AJ, Steiger P, et al. Endothelial cell infection and endotheliitis in COVID-19. Lancet 2020;395(10234):1417-1418

21 Favaloro EJ, Lippi G. Recommendations for minimal laboratory testing panels in patients with COVID-19: potential for prognostic monitoring. Semin Thromb Hemost 2020;46(03):379-382

22 Christensen B, Favaloro EJ, Lippi G, Van Cott EM. Hematology laboratory abnormalities in patients with coronavirus disease 2019 (COVID-19). Semin Thromb Hemost 2020;46(07):845-849

23 Levi M, Thachil J. Coronavirus disease 2019 coagulopathy: disseminated intravascular coagulation and thrombotic microangiopathy-either, neither, or both. Semin Thromb Hemost 2020;46 (07):781-784

24 Thachil J, Srivastava A. SARS-2 Coronavirus-Associated Hemostatic Lung Abnormality In COVID-19: is it pulmonary thrombosis or pulmonary embolism? Semin Thromb Hemost 2020;46(07): $777-780$

25 Schulman S. Coronavirus disease 2019, prothrombotic factors, and venous thromboembolism. Semin Thromb Hemost 2020;46 (07):772-776

26 Kwaan HC. Coronavirus disease 2019: the role of the fibrinolytic system from transmission to organ injury and sequelae. Semin Thromb Hemost 2020;46(07):841-844 
27 Larsen JB, Pasalic L, Hvas AM. Platelets in coronavirus disease 2019. Semin Thromb Hemost 2020;46(07):823-825

28 Favaloro EJ, Henry BM, Lippi G. Increased VWF and decreased ADAMTS13 in COVID-19: creating a milieu for (micro)thrombosis? Semin Thromb Hemost 2021; In press

29 Mendoza-Pinto C, Escárcega RO, García-Carrasco M, Bailey DJO, Gálvez-Romero JL, Cervera R. Viral infections and their relationship with catastrophic antiphospholipid syndrome: a possible pathogenic mechanism of severe COVID-19 thrombotic complications. J Intern Med 2020;288(06):737-739

30 El Hasbani G, Taher AT, Jawad A, Uthman I. COVID-19, antiphospholipid antibodies, and catastrophic antiphospholipid syndrome: a possible association? Clin Med Insights Arthritis Musculoskelet Disord 2020;13:1179544120978667

31 Previtali G, Seghezzi M, Moioli V, et al. The pathogenesis of thromboembolic disease in covid-19 patients: could be a catastrophic antiphospholipid syndrome? Thromb Res 2020; 194:192-194

32 Zhang Y, Xiao M, Zhang S, et al. Coagulopathy and antiphospholipid antibodies in patients with Covid-19. N Engl J Med 2020;382 (17):e38

33 Metjian A, Lim W. ASH evidence-based guidelines: should asymptomatic patients with antiphospholipid antibodies receive primary prophylaxis to prevent thrombosis? Hematology (Am Soc Hematol Educ Program) 2009:247-249

34 Mustonen P, Lehtonen KV, Javela K, Puurunen M. Persistent antiphospholipid antibody (aPL) in asymptomatic carriers as a risk factor for future thrombotic events: a nationwide prospective study. Lupus 2014;23(14):1468-1476

35 Pengo V, Ruffatti A, Legnani C, et al. Incidence of a first thromboembolic event in asymptomatic carriers of high-risk antiphospholipid antibody profile: a multicenter prospective study. Blood 2011;118(17):4714-4718

36 Yelnik CM, Urbanski G, Drumez E, et al. Persistent triple antiphospholipid antibody positivity as a strong risk factor of first thrombosis, in a long-term follow-up study of patients without history of thrombosis or obstetrical morbidity. Lupus 2017;26 (02):163-169

37 Devreese KMJ, de Groot PG, de Laat B, et al. Guidance from the Scientific and Standardization Committee for lupus anticoagulant/antiphospholipid antibodies of the International Society on Thrombosis and Haemostasis: update of the guidelines for lupus anticoagulant detection and interpretation. J Thromb Haemost 2020;18(11):2828-2839

38 Keeling D, Mackie I, Moore GW, Greer IA, Greaves MBritish Committee for Standards in Haematology. Guidelines on the investigation and management of antiphospholipid syndrome. Br J Haematol 2012;157(01):47-58

39 Ledford-Kraemer M, Moore GW, Bottenus R, et al. Clinical and Laboratory Standards Institute (CLSI). Laboratory Testing for the Lupus Anticoagulant; Approved Guideline. CLSI document H60-A Wayne, PA: CLSI; 2014

40 Yasri S, Wiwanitkit V. COVID-19, antiphospholipid syndrome and thrombosis. Clin Appl Thromb Hemost 2020;26:1076029620931927

41 Lerma LA, Chaudhary A, Bryan A, Morishima C, Wener MH, Fink SL. Prevalence of autoantibody responses in acute coronavirus disease 2019 (COVID-19). J Transl Autoimmun 2020;3:100073

42 Galeano-Valle F, Oblitas CM, Ferreiro-Mazón MM, et al. Antiphospholipid antibodies are not elevated in patients with severe COVID-19 pneumonia and venous thromboembolism. Thromb Res 2020;192:113-115

43 Gatto M, Perricone C, Tonello M, et al. Frequency and clinical correlates of antiphospholipid antibodies arising in patients with SARS-CoV-2 infection: findings from a multicentre study on 122 cases. Clin Exp Rheumatol 2020;38(04):754-759

44 Siguret V, Voicu S, Neuwirth M, et al. Are antiphospholipid antibodies associated with thrombotic complications in critically ill COVID-19 patients? Thromb Res 2020;195:74-76
45 Fan S, Xiao M, Han F, et al. Neurological manifestations in critically ill patients with COVID-19: a retrospective study. Front Neurol 2020;11:806

46 Pineton de Chambrun M, Frere C, Miyara M, et al. High frequency of antiphospholipid antibodies in critically ill COVID-19 patients: a link with hypercoagulability? J Intern Med 2021;289(03): 422-424

47 Popovic B, Varlot J, Metzdorf PA, Jeulin H, Goehringer F, Camenzind $\mathrm{E}$. Changes in characteristics and management among patients with ST-elevation myocardial infarction due to COVID-19 infection. Catheter Cardiovasc Interv 2021;97(03): E319-E326

48 Rothstein A, Oldridge O, Schwennesen H, Do D, Cucchiara BL. Acute cerebrovascular events in hospitalized COVID-19 patients. Stroke 2020;51(09):e219-e222

49 Devreese KMJ, Linskens EA, Benoit D, Peperstraete H. Antiphospholipid antibodies in patients with COVID-19: a relevant observation? J Thromb Haemost 2020;18(09):2191-2201

50 Reyes Gil M, Barouqa M, Szymanski J, Gonzalez-Lugo JD, Rahman S, Billett HH. Assessment of lupus anticoagulant positivity in patients with coronavirus disease 2019 (COVID-19). JAMA Netw Open 2020;3(08):e2017539

51 Amezcua-Guerra LM, Rojas-Velasco G, Brianza-Padilla M, et al. Presence of antiphospholipid antibodies in COVID-19: case series study. Ann Rheum Dis. 2020:annrheumdis-2020-218100

52 de Ocáriz XGL, Quismondo NC, Guerrero EV, Rodríguez MR, Díaz RA, López JM. Thrombosis and antiphospholipid antibodies in patients with SARS-COV-2 infection (COVID-19). Int J Lab Hematol 2020;42(06):e280-e282

53 Cuenca Saez MA, Gomez-Biezna SL. Immunoglobulin A antiphospholipid antibodies in patients with chilblain-like lesions during the COVID-19 pandemic. Actas Dermosifiliogr 2021;112(03): 290-292

54 Tvito A, Ben-Chetrit E, Zimmerman FS, Asher E, Helviz Y. Lupus anticoagulant in patients with COVID-19. Int J Lab Hematol 2021; 43(01):e17-e18

55 Bertin D, Brodovitch A, Beziane A, et al. Anticardiolipin IgG autoantibody level is an independent risk factor for COVID-19 severity. Arthritis Rheumatol 2020;72(11):1953-1955

56 Ferrari E, Sartre B, Squara F, et al. High prevalence of acquired thrombophilia without prognosis value in patients with coronavirus disease 2019. J Am Heart Assoc 2020;9(21):e017773

57 Pascolini S, Vannini A, Deleonardi G, et al. COVID-19 and immunological dysregulation: can autoantibodies be useful? Clin Transl Sci 2021;14(02):502-508

58 Hasan Ali O, Bomze D, Risch L, et al. Severe COVID-19 is associated with elevated serum IgA and antiphospholipid IgA-antibodies. Clin Infect Dis 2020 (ePub ahead of print). Doi: 10.1093/cid/ ciaa1496

59 Zhang Y, Cao W, Jiang W, et al. Profile of natural anticoagulant, coagulant factor and anti-phospholipid antibody in critically ill COVID-19 patients. J Thromb Thrombolysis 2020;50(03): 580-586

60 Tan YK, Goh C, Leow AST, et al. COVID-19 and ischemic stroke: a systematic review and meta-summary of the literature. J Thromb Thrombolysis 2020;50(03):587-595

61 Lee KW, Yusof Khan AHK, Ching SM, et al. Stroke and novel coronavirus infection in humans: a systematic review and meta-analysis. Front Neurol 2020;11:579070

$62 \mathrm{Tu}$ TM, Goh C, Tan YK, et al. Cerebral venous thrombosis in patients with COVID-19 infection: a case series and systematic review. J Stroke Cerebrovasc Dis 2020;29(12):105379

63 Xiao M, Zhang Y, Zhang S, et al. Antiphospholipid antibodies in critically ill patients with COVID-19. Arthritis Rheumatol 2020;72 (12):1998-2004

64 Borghi MO, Beltagy A, Garrafa E, et al. Anti-phospholipid antibodies in COVID-19 are different from those detectable in the anti-phospholipid syndrome. Front Immunol 2020;11:584241 
65 Woodruff MC, Ramonell RP, Lee FE, Sanz I. Broadly-targeted autoreactivity is common in severe SARS-CoV-2 infection. [preprint]. 2020 Doi: 10.1101/2020.10.21.20216192

66 Fan BE, Ng J, Chan SSW, et al. COVID-19 associated coagulopathy in critically ill patients: a hypercoagulable state demonstrated by parameters of haemostasis and clot waveform analysis. J Thromb Thrombolysis 2021;51(03):663-674

67 Zuo Y, Estes SK, Ali RA, et al. Prothrombotic autoantibodies in serum from patients hospitalized with COVID-19. Sci Transl Med 2020;12(570):eabd3876

68 Gazzaruso C, Mariani G, Ravetto C, Malinverni L, Tondelli E, Cerrone M, Sala V, Bevilacqua L, Altavilla T, Coppola A, Gallotti P. Lupus anticoagulant and mortality in patients hospitalized for COVID-19. J Thromb Thrombolysis 2021;52:85-91

69 Le Joncour A, Frere C, Martin-Toutain I, et al. Antiphospholipid antibodies and thrombotic events in COVID-19 patients hospitalized in medicine ward. Autoimmun Rev 2021;20(02):102729

70 Novelli L, Motta F, De Santis M, Ansari AA, Gershwin ME, Selmi C. The JANUS of chronic inflammatory and autoimmune diseases onset during COVID-19 - a systematic review of the literature. J Autoimmun 2021;117:102592

71 Frapard T, Hue S, Rial C, de Prost N, Mekontso Dessap A. Antiphospholipid autoantibodies and thrombosis in patients with COVID-19: comment on the article by Bertin et al. Arthritis Rheumatol 2021;73(05):897-899

72 Cristiano A, Fortunati V, Cherubini F, Bernardini S, Nuccetelli M. Anti-phospholipids antibodies and immune complexes in COVID19 patients: a putative role in disease course for anti-annexin-V antibodies. Clin Rheumatol 2021 (epub ahead of print). Doi: $10.1007 / \mathrm{s} 10067-021-05580-3$
73 Shi H, Zuo Y, Gandhi AA, et al. Endothelial cell-activating antibodies in COVID-19. [preprint]. 2021 Doi: 10.1101/2021.01.18.21250041

74 Hamadé A, Woehl B, Harzallah I, Talbot M, Tousch J, Jambert L. Antiphospholipid antibodies in patients with coronavirus disease 2019 infection hospitalized in conventional unit. Blood Coagul Fibrinolysis 2021;32(02):73-79

75 Karahan S, Erol K, Yuksel RC, Artan C, Celik I. Antiphospholipid antibodies in COVID-19-associated pneumonia patients in intensive care unit. Mod Rheumatol 2021 (ePub ahead of print). Doi: 10.1080/14397595.2021.1892257

76 Beyrouti R, Adams ME, Benjamin L, et al. Characteristics of ischaemic stroke associated with COVID-19. J Neurol Neurosurg Psychiatry 2020;91(08):889-891

77 Abdel-Wahab N, Talathi S, Lopez-Olivo MA, Suarez-Almazor ME. Risk of developing antiphospholipid antibodies following viral infection: a systematic review and meta-analysis. Lupus 2018;27 (04):572-583

78 Abdel-Wahab N, Lopez-Olivo MA, Pinto-Patarroyo GP, SuarezAlmazor ME. Systematic review of case reports of antiphospholipid syndrome following infection. Lupus 2016;25(14):1520-1531

79 Cavalli E, Bramanti A, Ciurleo R, et al. Entangling COVID-19 associated thrombosis into a secondary antiphospholipid antibody syndrome: diagnostic and therapeutic perspectives (Review). Int J Mol Med 2020;46(03):903-912

80 Million M, Bardin N, Bessis S, et al. Thrombosis and antiphospholipid antibody syndrome during acute $\mathrm{Q}$ fever: a cross-sectional study. Medicine (Baltimore) 2017;96(29):e7578

81 Mendoza-Pinto C, García-Carrasco M, Cervera R. Role of infectious diseases in the antiphospholipid syndrome (including its catastrophic variant). Curr Rheumatol Rep 2018;20(10):62 